\title{
A Refinement of the Local Serrin-Type Regularity Criterion for a Suitable Weak Solution to the Navier-Stokes Equations
}

\author{
Jiří Neustupa
}

\begin{abstract}
We formulate a new criterion for regularity of a suitable weak solution $\mathbf{v}$ to the NavierStokes equations at the space-time point $\left(\mathrm{x}_{0}, t_{0}\right)$. The criterion imposes a Serrin-type integrability condition on $\mathbf{v}$ only in a backward neighbourhood of $\left(\mathbf{x}_{0}, t_{0}\right)$, intersected with the exterior of a certain space-time paraboloid with vertex at point $\left(\mathrm{x}_{0}, t_{0}\right)$. We make no special assumptions on the solution in the interior of the paraboloid.
\end{abstract}

AMS math. classification (2000): 35Q30, 76D03, 76D05.

Keywords: Navier-Stokes equations, suitable weak solution, regularity.

\section{Introduction}

The Navier-Stokes system. Let $\Omega$ be a domain in $\mathbb{R}^{3}$ and $T>0$. Put $Q_{T}:=\Omega \times(0, T)$. We deal with the Navier-Stokes system

$$
\begin{aligned}
\partial_{t} \mathbf{v}+\mathbf{v} \cdot \nabla \mathbf{v} & =-\nabla p+\nu \Delta \mathbf{v} & & \text { in } Q_{T}, \\
\operatorname{div} \mathbf{v} & =0 & & \text { in } Q_{T}
\end{aligned}
$$

for the unknown velocity $\mathbf{v}=\left(v_{1}, v_{2}, v_{3}\right)$ and pressure $p$. Symbol $\nu$ denotes the coefficient of viscosity. It is a positive constant.

Notation. Vector functions and spaces of vector functions are denoted by boldface letters. The norm of a scalar- or vector- or tensor-valued function with components in $L^{q}(\Omega)$ (respectively $W^{k, q}(\Omega)$ ) is denoted by $\|\cdot\|_{q ; \Omega}$ (respectively $\|\cdot\|_{k, q ; \Omega}$ ). Norms in spaces of functions on other domains than $\Omega$ are denoted by analogy.

Weak and suitable weak solution, regular and singular points. The definition of a weak solution to the system (1.1), (1.2) is explained, together with basic properties of weak solutions, e.g. in the books by O. A. Ladyzhenskaya [5], R. Temam [18], H. Sohr [15] and in the survey paper [3] by G. P. Galdi. Here, we only recall that weak solution $\mathbf{v}$ of (1.1), (1.2) belongs to $L^{\infty}\left(0, T ; \mathbf{L}^{2}(\Omega)\right) \cap L^{2}\left(0, T ; \mathbf{W}^{1,2}(\Omega)\right)$. While the existence of a weak solution (satisfying various kinds of boundary conditions) is known on an arbitrarily long time interval $(0, T)$, its regularity is generally an open problem.

By the definition from paper [1] by L. Caffarelli, R. Kohn and L. Nirenberg, the point $\left(\mathbf{x}_{0}, t_{0}\right) \in$ $Q_{T}$ is said to be a regular point of weak solution $\mathbf{v}$ if there exists a neighborhood $U$ of $\left(\mathbf{x}_{0}, t_{0}\right)$ 
such that $\mathbf{v} \in \mathbf{L}^{\infty}(U)$. Points in $Q_{T}$ that are not regular are called singular. A weak solution $\mathbf{v}$ of system (1.1), (1.2) is called a suitable weak solution if an associated pressure $p$ belongs to $L^{5 / 4}\left(Q_{T}\right)$ and the pair $(\mathbf{v}, p)$ satisfies the so called generalized energy inequality

$$
2 \nu \int_{0}^{T} \int_{\Omega}|\nabla \mathbf{v}|^{2} \phi \mathrm{d} \mathbf{x} \mathrm{d} t \leq \int_{0}^{T} \int_{\Omega}\left[|\mathbf{v}|^{2}\left(\partial_{t} \phi+\nu \Delta \phi\right)+\left(|\mathbf{v}|^{2}+2 p\right) \mathbf{v} \cdot \nabla \phi\right] \mathrm{d} \mathbf{x} \mathrm{d} t
$$

for every non-negative function $\phi$ from $C_{0}^{\infty}\left(Q_{T}\right)$. It is also shown in [1] that the set of singular points of suitable weak solution $\mathbf{v}$ has the 1 -dimensional parabolic measure (which dominates the 1-dimensional Hausdorff measure) equal to zero. This result follows (by a standard covering procedure) from the local regularity criterion (also proven in [1]), saying that there exists a universal constant $\epsilon>0$ such that if

$$
\limsup _{\delta \rightarrow 0+} \frac{1}{\delta} \int_{t_{0}-7 \delta^{2} / 8}^{t_{0}+\delta^{2} / 8} \int_{B_{\delta}\left(\mathbf{x}_{0}\right)}|\nabla \mathbf{v}|^{2} \mathrm{~d} \mathbf{x} \mathrm{d} t \leq \epsilon
$$

then $\left(\mathbf{x}_{0}, t_{0}\right)$ is a regular point of $\mathbf{v}$. Analogous results and some generalizations can also be found in papers [6], [7], [4], [19], and others.

Some other local regularity criteria. The next criteria are often called $\epsilon$-criteria because they state that there exists a universal constant $\epsilon>0$ (sufficiently small) such that if a certain quantity is less than or equal to $\epsilon$ then $\left(\mathbf{x}_{0}, t_{0}\right)$ is a regular point of solution $\mathbf{v}$. Constant $\epsilon$ is generally different in different criteria.

F. Lin [7] proved that the condition

$$
\lim _{\delta \rightarrow 0+} \frac{1}{\delta^{2}} \int_{t_{0}-\delta^{2}}^{t_{0}} \int_{B_{\delta}\left(\mathbf{x}_{0}\right)}\left(|\mathbf{v}|^{3}+|p|^{\frac{3}{2}}\right) \mathrm{d} \mathbf{x} \mathrm{d} t \leq \epsilon
$$

guarantees that $\mathbf{v}$ is Hölder continuous in the set $\overline{B_{\rho}\left(\mathbf{x}_{0}\right)} \times\left[t_{0}-\rho^{2}, t_{0}\right]$ (for some $\rho>0$ ), which implies that $\left(\mathbf{x}_{0}, t_{0}\right)$ is a regular point of solution $\mathbf{v}$. Lin's criterion has been several times improved (see [8], [13] and [20]). Wolf's criterion (see [20]) says that if $3 \leq r, s \leq \infty$ and

$$
\delta^{s\left[1-\left(\frac{2}{r}+\frac{3}{s}\right)\right]} \int_{t_{0}-\delta^{2}}^{t_{0}}\left(\int_{B_{\delta}\left(\mathbf{x}_{0}\right)}|\mathbf{v}|^{s} \mathrm{~d} \mathbf{x}\right)^{\frac{r}{s}} \mathrm{~d} t \leq \epsilon
$$

holds for at least one $\delta>0$ then $\mathbf{v}$ is Hölder continuous in the set $\overline{B_{\delta / 2}\left(\mathbf{x}_{0}\right)} \times\left[t_{0}-\delta^{2} / 4, t_{0}\right]$. Particularly, if we choose $r=s=3$ then we observe that if the inequality

$$
\frac{1}{\delta^{2}} \int_{t_{0}-\delta^{2}}^{t_{0}} \int_{B_{\delta}\left(\mathbf{x}_{0}\right)}|\mathbf{v}|^{3} \mathrm{~d} \mathbf{x} \mathrm{d} t \leq \epsilon
$$

holds for at least one $\delta>0$ then $\left(\mathbf{x}_{0}, t_{0}\right)$ is a regular point of solution $\mathbf{v}$.

A series of other local regularity criteria can be found in [14], [9], [2], etc.

Let us finally recall that $\mathrm{S}$. Takahashi [16] proved that if the norm of a weak solution $\mathbf{v}$ in $L_{w}^{r}\left(t_{0}-\rho^{2}, t_{0} ; \mathbf{L}^{s}\left(B_{\rho}\left(\mathbf{x}_{0}\right)\right)\right.$ (where $L_{w}^{r}$ denotes the weak $L^{r}$-space and $2 / r+3 / s \leq 1,3<s \leq$ $\infty)$ is less than or equal to $\epsilon$ then $\left(\mathbf{x}_{0}, t_{0}\right)$ is a regular point of $\mathbf{v}$.

Takahashi's criterion has been modified in paper [10], where $\mathbf{v}$ is supposed to be integrable with powers $r \in[3, \infty)$ (in time) and $s \in(3, \infty)$ (in space) not necessarily in the whole backward 
parabolic neighbourhood $Q_{a, \rho}:=B_{\sqrt{a} \rho}\left(\mathbf{x}_{0}\right) \times\left(t_{0}-\rho^{2}, t_{0}\right)$, but only in the intersection of this neighbourhood with the exterior of the space-time paraboloid

$$
P_{a}: \quad \sqrt{a\left(t_{0}-t\right)}=\left|\mathbf{x}-\mathbf{x}_{0}\right|
$$

(with vertex at $\left(\mathbf{x}_{0}, t_{0}\right)$ ). Exponents $r$ and $s$ are required to satisfy the condition $2 / r+3 / s<1$, and number $a$ is supposed to satisfy the inequality $0<a<4 \nu$ in [10]. Moreover, it is also supposed in [10] that there exist real numbers $R$ and $h$ such that $R>1,0<h<R-1$ and

$$
\int_{t_{0}-\rho^{2} / R^{2}}^{t_{0}}\left(\int_{(R-h) \sqrt{a\left(t_{0}-t\right)}<\left|\mathbf{x}-\mathbf{x}_{0}\right|<R \sqrt{a\left(t_{0}-t\right)}}|p(\mathbf{x}, t)|^{\beta} \mathrm{d} \mathbf{x}\right)^{\alpha / \beta} \mathrm{d} t<\infty
$$

for $\alpha \in\left[\frac{r}{r-1}, \infty\right)$ and $\beta \in\left(\frac{3}{2}, \infty\right)$, satisfying the inequality $2 / \alpha+3 / \beta<2$. Note that the domain of the integral in (1.6) is the exterior of paraboloid $P_{a}$, intersected with neighbourhood $Q_{a, \rho}$.

On the result of this paper. In this paper, we improve the regularity criterion from [10] especially so that we remove the assumption on the pressure. Concretely, we show that condition (1.6) can be omitted. Moreover, in comparison to [10], we assume that $2 / r+3 / s=1$ and we also use a weaker restriction on parameter $a$ (see Theorem 1). This is enabled by finer estimates in Sections 3, 5, and by a different treatment of the term containing the transformed pressure $p^{\prime}$ in Section 3 , see Lemma2.

Our Theorem 1(formulated below) imposes the Serrin-type condition only on velocity $\mathbf{v}$ in an arbitrarily small region $U_{a, \rho}$ in $Q_{T}$, which is defined as follows:

$$
U_{a, \rho}:=\left\{(\mathbf{x}, t) \in Q_{T} ; t_{0}-\rho^{2}<t<t_{0} \text { and } \sqrt{a\left(t_{0}-t\right)}<\left|\mathbf{x}-\mathbf{x}_{0}\right|<\sqrt{a} \rho\right\}
$$

(for $a>0$ and $0<\rho<\sqrt{t_{0}}$ ). In contrast to a series of other regularity criteria, we make no assumptions on $\mathbf{v}$ or $p$ in the interior of paraboloid $P_{a}$, concretely in set $V_{a, \rho}$ which is the interior of $P_{a}$, intersected with neighbourhood $Q_{a, \rho}$.

A generalization of Theorem 1 where parameter $a$ does not appear, is presented in Section 6

Sets $U_{a, \rho}$ and $V_{a, \rho}$ are sketched on Fig. 1. They are separated by paraboloid $P_{a}$.
Fig. 1:

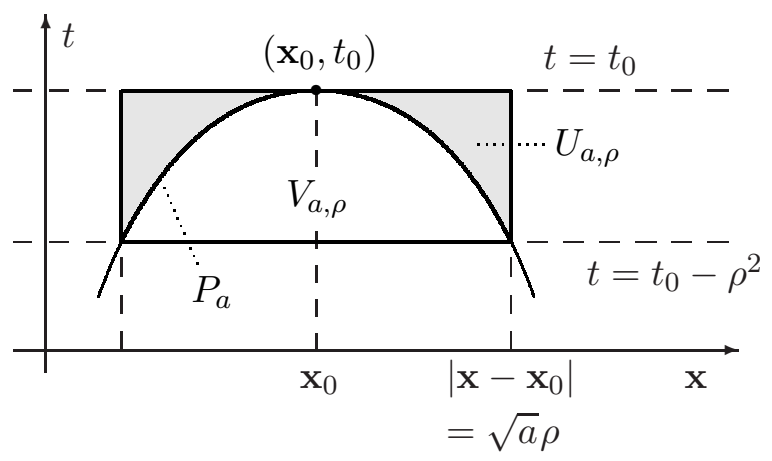

We denote by $\lambda_{S}\left(B_{1}\right)$ be the least eigenvalue of the Dirichlet-Stokes operator in the unit ball $B_{1}$ in $\mathbb{R}^{3}$. Note that the question of how to calculate explicitly the eigenvalues and eigenfunctions of the Stokes operator in the ball was asked by O. A. Ladyzhenskaya in 2003. It can be deduced from [11] that $\lambda_{S}\left(B_{1}\right) \leq \mu_{1}^{2}\left(J_{3 / 2}\right)$, where $\mu_{1}\left(J_{3 / 2}\right) \doteq 4.4934$ is the first positive root of the Bessel function $J_{3 / 2}$. Using the variational representation of $\lambda_{S}\left(B_{1}\right)$ (i.e. that $\lambda_{S}\left(B_{1}\right)$ equals the infimum 
of $\|\nabla \mathbf{u}\|_{2 ; B_{1}}^{2} /\|\mathbf{u}\|_{2 ; B_{1}}^{2}$ over all non-zero divergence-free functions $\left.\mathbf{u} \in \mathbf{W}_{0}^{1,2}\left(B_{1}\right)\right)$ and the analogous representation of $\lambda_{L}\left(B_{1}\right)$ (the principal eigenvalue of the Dirichlet-Laplacian on the unit ball), we obtain $\lambda_{S}\left(B_{1}\right) \geq \lambda_{L}\left(B_{1}\right)$. The latter equals $\pi^{2}$, see e.g. [11].

The main result of this paper says:

Theorem 1. Let $\mathbf{v}$ be a suitable weak solution of system (1.1), (1.2), $\left(\mathbf{x}_{0}, t_{0}\right) \in Q_{T}$ and $0<$ $a<4 \nu \lambda_{S}\left(B_{1}\right)$. Let $\rho>0$ be so small that $Q_{a, \rho} \subset Q_{T}$. Suppose that function $\mathbf{v}$ satisfies the integrability condition in set $U_{a, \rho}$ :

$$
\int_{t_{0}-\rho^{2}}^{t_{0}}\left(\int_{\sqrt{a\left(t_{0}-t\right)}<\left|\mathbf{x}-\mathbf{x}_{0}\right|<\sqrt{a} \rho}|\mathbf{v}(\mathbf{x}, t)|^{s} \mathrm{~d} \mathbf{x}\right)^{\frac{r}{s}} \mathrm{~d} t<\infty
$$

for some $r, s$, satisfying the inequalities

$$
3 \leq r<\infty, \quad 3<s<\infty, \quad \frac{2}{r}+\frac{3}{s}=1 .
$$

Then $\left(\mathbf{x}_{0}, t_{0}\right)$ is a regular point of solution $\mathbf{v}$.

Theorem 1 shows that if a singularity eventually appears in a suitable weak solution of the Navier-Stokes system (1.1), (1.2) at the point $\left(\mathrm{x}_{0}, t_{0}\right)$ then it cannot develop only around point $\mathrm{x}_{0}$ itself (i.e. only in set $V_{a, \rho}$ ). On the other hand, "large" values of velocity must also be necessarily transferred to the point $\mathbf{x}_{0}$ from the sides at times $t<t_{0}$ with the speed increasing to infinity as $t \rightarrow t_{0}-$.

\section{Proof of Theorem 1- part I}

Notation and the used regularity criterion. We denote

$$
\theta(t):=\sqrt{a\left(t_{0}-t\right)} \quad \text { and } \quad G(\delta):=\frac{1}{\delta^{2}} \int_{t_{0}-\delta^{2}}^{t_{0}} \int_{\left|\mathbf{x}-\mathbf{x}_{0}\right|<\sqrt{a} \delta}|\mathbf{v}|^{3} \mathrm{~d} \mathbf{x} \mathrm{d} t .
$$

We split $G(\delta)$ to two parts:

$$
G(\delta)=G^{I}(\delta)+G^{I I}(\delta)
$$

where

$$
\begin{aligned}
G^{I}(\delta) & :=\frac{1}{\delta^{2}} \int_{t_{0}-\delta^{2}}^{t_{0}} \int_{\theta(t)<\left|\mathbf{x}-\mathbf{x}_{0}\right|<\sqrt{a} \delta}|\mathbf{v}|^{3} \mathrm{~d} \mathbf{x} \mathrm{d} t \\
G^{I I}(\delta) & :=\frac{1}{\delta^{2}} \int_{t_{0}-\delta^{2}}^{t_{0}} \int_{\left|\mathbf{x}-\mathbf{x}_{0}\right|<\theta(t)}|\mathbf{v}|^{3} \mathrm{~d} \mathbf{x} \mathrm{d} t .
\end{aligned}
$$

We will show that

$$
\lim _{\delta \rightarrow 0+} \inf _{\delta} G(\delta)=0 .
$$

Since (2.3) implies the validity of condition (1.4), it also implies that $\left(\mathbf{x}_{0}, t_{0}\right)$ is a regular point of solution $\mathbf{v}$.

An estimate of $G^{I}(\delta)$. Assume that $r>3$. Then $G^{I}(\delta)$ can be estimated as follows:

$$
G^{I}(\delta) \leq \frac{1}{\delta^{2}} \int_{t_{0}-\delta^{2}}^{t_{0}}\left(\int_{\theta(t)<\left|\mathbf{x}-\mathbf{x}_{0}\right|<\sqrt{a} \delta}|\mathbf{v}|^{s} \mathrm{~d} \mathbf{x}\right)^{\frac{3}{s}}\left(\frac{4 \pi(\sqrt{a} \delta)^{3}}{3}\right)^{1-\frac{3}{s}} \mathrm{~d} t
$$




$$
\leq\left[\frac{4 \pi a^{\frac{3}{2}}}{3}\right]^{1-\frac{3}{s}}\left[\int_{t_{0}-\delta^{2}}^{t_{0}}\left(\int_{\theta(t)<\left|\mathbf{x}-\mathbf{x}_{0}\right|<\sqrt{a} \delta}|\mathbf{v}|^{s} \mathrm{~d} \mathbf{x}\right)^{\frac{r}{s}} \mathrm{~d} t\right]^{\frac{3}{r}} .
$$

This implies, due to conditions (1.7) and (1.8), that

$$
\lim _{\delta \rightarrow 0+} G^{I}(\delta)=0 .
$$

We obtain the same information in the case $r=3$, too.

Transformation to the new coordinates $\mathbf{x}^{\prime}, t^{\prime}$. In order to estimate $G^{I I}(\delta)$, we transform the integral in (2.2) and the system (1.1), (1.2) to the new coordinates $\mathrm{x}^{\prime}$ and $t^{\prime}$, which are related to $\mathrm{x}$ and $t$ through the formulas

$$
\mathbf{x}^{\prime}=\frac{\mathbf{x}-\mathbf{x}_{0}}{\theta(t)}, \quad t^{\prime}=\int_{t_{0}-\rho^{2}}^{t} \frac{\mathrm{d} s}{\theta^{2}(s)}=\frac{1}{a} \ln \frac{\rho^{2}}{t_{0}-t} .
$$

Then

$$
t=t_{0}-\rho^{2} \mathrm{e}^{-a t^{\prime}} \quad \text { and } \quad \theta(t)=\sqrt{a} \rho \mathrm{e}^{-\frac{1}{2} a t^{\prime}} .
$$

Fig. 2:

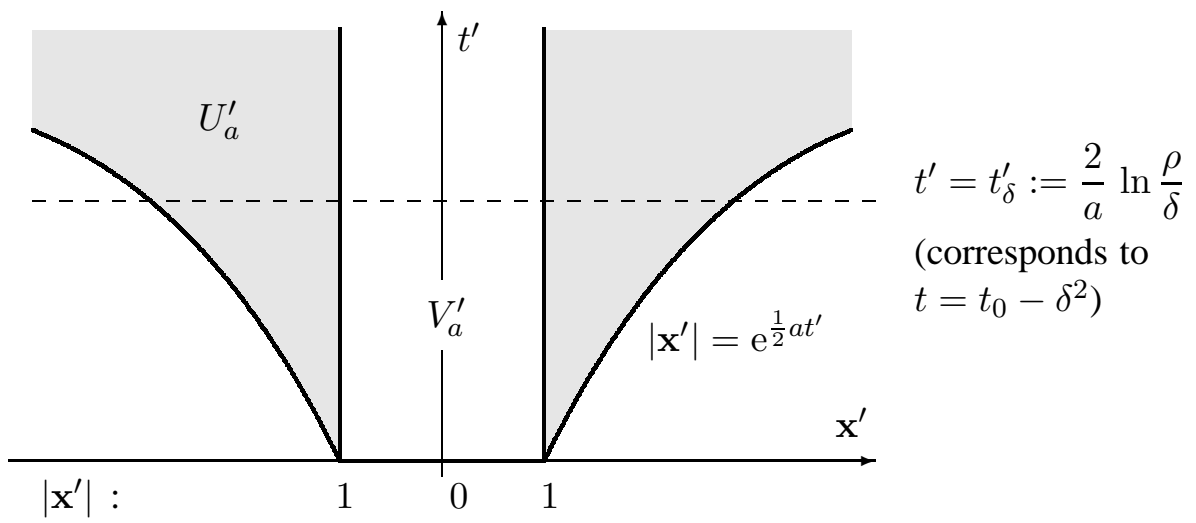

The time interval $\left(t_{0}-\rho^{2}, t_{0}\right)$ on the $t$-axis now corresponds to the interval $(0, \infty)$ on the $t^{\prime}-$ axis. Equations (2.5) represent a one-to-one transformation of the parabolic region $V_{a, \rho}$ in the $\mathbf{x}, t$-space onto the infinite stripe

$$
V_{a}^{\prime}:=\left\{\left(\mathbf{x}^{\prime}, t^{\prime}\right) \in \mathbb{R}^{4} ; t^{\prime}>0 \text { and }\left|\mathbf{x}^{\prime}\right|<1\right\}
$$

in the $\mathbf{x}^{\prime}, t^{\prime}$-space. Similarly, (2.5) is a one-to-one transformation of set $U_{a, \rho}$ in the $\mathbf{x}, t$-space onto

$$
U_{a}^{\prime}:=\left\{\left(\mathbf{x}^{\prime}, t^{\prime}\right) \in \mathbb{R}^{4} ; t^{\prime}>0 \text { and } 1<\left|\mathbf{x}^{\prime}\right|<\mathrm{e}^{\frac{1}{2} a t^{\prime}}\right\}
$$

in the $\mathbf{x}^{\prime}, t^{\prime}$-space. We denote

$$
t_{\delta}^{\prime}:=\frac{2}{a} \ln \frac{\rho}{\delta} .
$$

Then $t^{\prime}=t_{\delta}^{\prime}$ corresponds to $t=t_{0}-\delta^{2}$. Obviously, $\mathrm{e}^{-\frac{1}{2} a t_{\delta}^{\prime}}=\delta / \rho$ and $\delta \rightarrow 0+$ corresponds to $t_{\delta}^{\prime} \rightarrow \infty$. If we put

$$
\begin{aligned}
& \mathbf{v}(\mathbf{x}, t)=\frac{1}{\theta(t)} \mathbf{v}^{\prime}\left(\frac{\mathbf{x}-\mathbf{x}_{0}}{\theta(t)}, \frac{1}{a} \ln \frac{\rho^{2}}{t_{0}-t}\right) \\
& p(\mathbf{x}, t)=\frac{1}{\theta^{2}(t)} p^{\prime}\left(\frac{\mathbf{x}-\mathbf{x}_{0}}{\theta(t)}, \frac{1}{a} \ln \frac{\rho^{2}}{t_{0}-t}\right)
\end{aligned}
$$


then functions $\mathbf{v}^{\prime}, p^{\prime}$ represent a suitable weak solution of the system of equations

$$
\begin{aligned}
\partial_{t^{\prime}} \mathbf{v}^{\prime}+\mathbf{v}^{\prime} \cdot \nabla^{\prime} \mathbf{v}^{\prime} & =-\nabla^{\prime} p^{\prime}+\nu \Delta^{\prime} \mathbf{v}^{\prime}-\frac{1}{2} a \mathbf{v}^{\prime}-\frac{1}{2} a \mathbf{x}^{\prime} \cdot \nabla^{\prime} \mathbf{v}^{\prime} \\
\operatorname{div}^{\prime} \mathbf{v}^{\prime} & =0
\end{aligned}
$$

in any bounded sub-domain of $Q_{a}^{\prime}:=\left\{\left(\mathbf{x}^{\prime}, t^{\prime}\right) \in \mathbb{R}^{4} ; t^{\prime}>0\right.$ and $\left.\left|\mathbf{x}^{\prime}\right|<\mathrm{e}^{\frac{1}{2} a t^{\prime}}\right\}$. (The symbols $\nabla^{\prime}$ and $\Delta^{\prime}$ denote the nabla operator and the Laplace operator with respect to the spatial variable $\mathbf{x}^{\prime}$.) Sets $A_{R_{1}, R_{2}}(t), A_{R_{1}, R_{2}}^{\prime}$ and $B_{R_{1}}^{\prime}$. Let $0<R_{1}<R_{2}$. We denote $A_{R_{1}, R_{2}}(t):=\{\mathrm{x} \in$ $\left.\mathbb{R}^{3} ; R_{1} \theta(t)<\left|\mathbf{x}-\mathbf{x}_{0}\right|<R_{2} \theta(t)\right\}$ and $A_{R_{1}, R_{2}}^{\prime}:=\left\{\mathbf{x}^{\prime} \in \mathbb{R}^{3} ; R_{1}<\left|\mathbf{x}^{\prime}\right|<R_{2}\right\}$. In order to keep a consistent notation, we also denote by $B_{R_{1}}^{\prime}$ the ball $\left\{\mathrm{x}^{\prime} \in \mathbb{R}^{3} ;\left|\mathrm{x}^{\prime}\right|<R_{1}\right\}$. The mapping $\mathbf{x} \mapsto \mathbf{x}^{\prime}=\left(\mathbf{x}-\mathbf{x}_{0}\right) / \theta(t)$ is a one-to-one transformation of $A_{R_{1}, R_{2}}(t)$ onto $A_{R_{1}, R_{2}}^{\prime}$ and $B_{R_{1} \theta(t)}\left(\mathbf{x}_{0}\right)$ onto $B_{R_{1}}^{\prime}$ at each time instant $t \in\left(t_{0}-\rho^{2}, t_{0}\right)$.

The first estimate of $G^{I I}(\delta)$. Recall that $t^{\prime}=t_{\delta}^{\prime}=2 a^{-1} \ln (\rho / \delta)$ corresponds to $t=t_{0}-\delta^{2}$ (see formulas (2.6) and (2.7)). Suppose that $\varphi$ is an infinitely differentiable function in $\mathbb{R}^{3}$ such that

$$
\varphi\left(\mathbf{x}^{\prime}\right) \begin{cases}=1 & \text { for }\left|\mathbf{x}^{\prime}\right| \leq 3 \\ \in[0,1] & \text { for } 3<\left|\mathbf{x}^{\prime}\right| \leq 4 \\ =0 & \text { for }\left|\mathbf{x}^{\prime}\right|>4\end{cases}
$$

Transforming $G^{I I}(\delta)$ to the variables $\mathbf{x}^{\prime}, t^{\prime}$, we get

$$
\begin{aligned}
G^{I I} & (\delta)=\frac{a \rho^{2}}{\delta^{2}} \int_{t_{\delta}^{\prime}}^{\infty} \int_{B_{1}^{\prime}}\left|\mathbf{v}^{\prime}\right|^{3} \mathrm{~d} \mathbf{x}^{\prime} \mathrm{e}^{-a t^{\prime}} \mathrm{d} t^{\prime} \leq \frac{a \rho^{2}}{\delta^{2}} \int_{t_{\delta}^{\prime}}^{\infty}\left\|\mathbf{v}^{\prime}\right\|_{6 ; B_{1}^{\prime}}^{\frac{3}{2}}\left\|\mathbf{v}^{\prime}\right\|_{2 ; B_{1}^{\prime}}^{\frac{3}{2}} \mathrm{e}^{-a t^{\prime}} \mathrm{d} t^{\prime} \\
& \leq \frac{a \rho^{2}}{\delta^{2}} \int_{t_{\delta}^{\prime}}^{\infty}\left\|\varphi \mathbf{v}^{\prime}\right\|_{6 ; B_{4}^{\prime}}^{\frac{3}{2}}\left\|\varphi \mathbf{v}^{\prime}\right\|_{2 ; B_{4}^{\prime}}^{\frac{3}{2}} \mathrm{e}^{-a t^{\prime}} \mathrm{d} t^{\prime} \\
& \leq \frac{2}{3^{\frac{3}{4}} \pi} \frac{a \rho^{2}}{\delta^{2}} \int_{t_{\delta}^{\prime}}^{\infty}\left\|\nabla^{\prime}\left(\varphi \mathbf{v}^{\prime}\right)\right\|_{2 ; B_{4}^{\prime}}^{\frac{3}{2}}\left\|\varphi \mathbf{v}^{\prime}\right\|_{2 ; B_{4}^{\prime}}^{\frac{3}{2}} \mathrm{e}^{-a t^{\prime}} \mathrm{d} t^{\prime} \\
& \leq \frac{2}{3^{\frac{3}{4}} \pi} \frac{a \rho^{2}}{\delta^{2}}\left(\int_{t_{\delta}^{\prime}}^{\infty}\left\|\nabla^{\prime}\left(\varphi \mathbf{v}^{\prime}\right)\right\|_{2 ; B_{4}^{\prime}}^{2} \mathrm{e}^{-\frac{2}{3} a t^{\prime}} \mathrm{d} t^{\prime}\right)^{\frac{3}{4}}\left(\int_{t_{\delta}^{\prime}}^{\infty}\left\|\varphi \mathbf{v}^{\prime}\right\|_{2 ; B_{4}^{\prime}}^{6} \mathrm{e}^{-2 a t^{\prime}} \mathrm{d} t^{\prime}\right)^{\frac{1}{4}} \\
& =\frac{2}{3^{\frac{3}{4}} \pi} a\left(\int_{t_{\delta}^{\prime}}^{\infty}\left\|\nabla^{\prime}\left(\varphi \mathbf{v}^{\prime}\right)\right\|_{2 ; B_{4}^{\prime}}^{2} \mathrm{e}^{-\frac{2}{3} a\left(t^{\prime}-t_{\delta}^{\prime}\right)} \mathrm{d} t^{\prime}\right)^{\frac{3}{4}} \\
& \cdot\left(\int_{t_{\delta}^{\prime}}^{\infty}\left\|\varphi \mathbf{v}^{\prime}\right\|_{2 ; B_{4}^{\prime}}^{6} \mathrm{e}^{-2 a\left(t^{\prime}-t_{\delta}^{\prime}\right)} \mathrm{d} t^{\prime}\right)^{\frac{1}{4}} .
\end{aligned}
$$

The factor $2 /\left(3^{\frac{3}{4}} \pi\right)$ comes from Sobolev's inequality, see e.g. [17, p. 34]. In order to estimate the integrals on the right hand side of (2.13), we use the next lemma and the generalized energy inequality in the $\mathbf{x}^{\prime}, t^{\prime}$-space.

Lemma 1. Assume that $0<\alpha \leq r, 0<\beta \leq s, R>1, t_{\delta}^{\prime}>2 a^{-1} \ln R$, and at least one of the two conditions

(a) $\quad \alpha=r, \omega \geq 0, \quad$ (b) $\quad \alpha<r, \omega>0$ 
holds. Then

$$
\int_{t_{\delta}^{\prime}}^{\infty}\left(\int_{A_{1, R}^{\prime}}\left|\mathbf{v}^{\prime}\right|^{\beta} \mathrm{d} \mathbf{x}^{\prime}\right)^{\frac{\alpha}{\beta}} \mathrm{e}^{-\omega a\left(t^{\prime}-t_{\delta}^{\prime}\right)} \mathrm{d} t^{\prime} \longrightarrow 0 \quad \text { as } t_{\delta}^{\prime} \rightarrow \infty .
$$

Proof. We use $C$ as a generic constant independent of $\delta$. In order to indicate that $C$ may depend on other quantities, we often write e.g. $C(R, a), C(R, \beta)$ or similar. We have

$$
\begin{gathered}
\int_{t_{\delta}^{\prime}}^{\infty}\left(\int_{A_{1, R}^{\prime}}\left|\mathbf{v}^{\prime}\right|^{\beta} \mathrm{d} \mathbf{x}^{\prime}\right)^{\frac{\alpha}{\beta}} \mathrm{e}^{-\omega a\left(t^{\prime}-t_{\delta}^{\prime}\right)} \mathrm{d} t^{\prime} \leq C(R, \beta) \int_{t_{\delta}^{\prime}}^{\infty}\left(\int_{A_{1, R}^{\prime}}\left|\mathbf{v}^{\prime}\right|^{s} \mathrm{~d} \mathbf{x}^{\prime}\right)^{\frac{\alpha}{s}} \mathrm{e}^{-\omega a\left(t^{\prime}-t_{\delta}^{\prime}\right)} \mathrm{d} t^{\prime} \\
=\frac{C(R, \beta, \rho)}{\delta^{2 \omega}} \int_{t_{0}-\delta^{2}}^{t_{0}}\left(\int_{A_{1, R}(t)}|\mathbf{v}|^{s} \mathrm{~d} \mathbf{x}\right)^{\frac{\alpha}{s}} \theta^{2 \omega+\alpha-3 \frac{\alpha}{s}-2}(t) \mathrm{d} t
\end{gathered}
$$

If condition (a) holds then the exponent $2 \omega+\alpha-3 \alpha / s-2$ equals $2 \omega+r(1-3 / s-2 / r)=2 \omega$. Hence the right hand side of 2.15 is less than or equal to

$$
C(R, \beta, \rho) \int_{t_{0}-\delta^{2}}^{t_{0}}\left(\int_{A_{1, R}(t)}|\mathbf{v}|^{s} \mathrm{~d} \mathbf{x}\right)^{\frac{r}{s}} \mathrm{~d} t .
$$

This tends to zero as $\delta \rightarrow 0+$ due to (1.7). If condition (b) holds then the right hand side of 2.15) is less than or equal to

$$
\frac{C(R, \beta, \rho)}{\delta^{2 \omega}}\left[\int_{t_{0}-\delta^{2}}^{t_{0}}\left(\int_{A_{1, R}(t)}|\mathbf{v}|^{s} \mathrm{~d} \mathbf{x}\right)^{\frac{r}{s}} \mathrm{~d} t\right]^{\frac{\alpha}{r}}\left[\int_{t_{0}-\delta^{2}}^{t_{0}} \theta^{\left[2 \omega+\alpha-3 \frac{\alpha}{s}-2\right] \frac{r}{r-\alpha}}(t) \mathrm{d} t\right]^{\frac{r-\alpha}{r}} .
$$

The last factor on the right hand side is

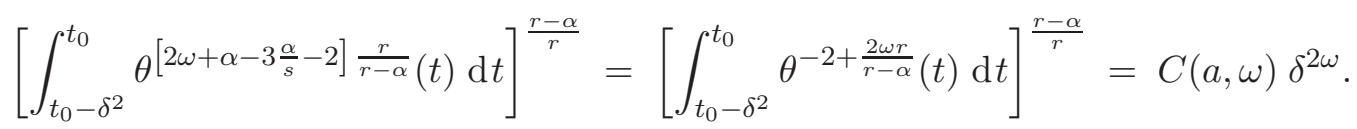

This shows that the right hand side of (2.15) tends to zero for $\delta \rightarrow 0+$ in the case of condition (b) as well. The proof is completed.

The generalized energy inequality in the $\mathbf{x}^{\prime}, t^{\prime}$-space. Since $\mathbf{v}^{\prime}, p^{\prime}$ is a suitable weak solution to the system (2.10), (2.11), it satisfies (by analogy with (1.3)) the generalized energy inequality

$$
\begin{array}{r}
2 \nu \int_{Q_{a}^{\prime}}\left|\nabla^{\prime} \mathbf{v}^{\prime}\right|^{2} \phi \mathrm{d} \mathbf{x}^{\prime} \mathrm{d} t^{\prime} \leq \int_{Q_{a}^{\prime}}[ \\
+\left.\mathbf{v}^{\prime}\right|^{2}\left(\partial_{t^{\prime}} \phi+\nu \Delta^{\prime} \phi\right)+\left(\left|\mathbf{v}^{\prime}\right|^{2}+2 p^{\prime}\right) \mathbf{v}^{\prime} \cdot \nabla^{\prime} \phi \\
\left.+\frac{1}{2} a\left|\mathbf{v}^{\prime}\right|^{2} \phi+\frac{1}{2} a\left(\mathbf{x}^{\prime} \cdot \nabla^{\prime} \phi\right)\left|\mathbf{v}^{\prime}\right|^{2}\right] \mathrm{d} \mathbf{x}^{\prime} \mathrm{d} t^{\prime}
\end{array}
$$

for every non-negative function $\phi$ from $C_{0}^{\infty}\left(Q_{a}^{\prime}\right)$.

Due to technical reasons, we further assume that $0<\delta<\rho / 4$. This assumption implies that $t_{\delta}^{\prime}>2 a^{-1} \ln 4$.

If function $\phi$ in inequality (2.16) has the form $\phi\left(\mathbf{x}^{\prime}, t^{\prime}\right)=\varphi\left(\mathbf{x}^{\prime}\right) \vartheta\left(t^{\prime}\right)$, where $\varphi$ is defined in (2.12) and $\vartheta$ is a $C^{\infty}$-function in $\left(2 a^{-1} \ln 4, \infty\right)$ with a compact support, we get

$$
2 \nu \int_{0}^{\infty} \int_{B_{4}^{\prime}}\left|\nabla^{\prime} \mathbf{v}^{\prime}\right|^{2} \varphi^{2}\left(\mathbf{x}^{\prime}\right) \vartheta\left(t^{\prime}\right) \mathrm{d} \mathbf{x}^{\prime} \mathrm{d} t^{\prime}
$$




$$
\begin{gathered}
\leq \int_{0}^{\infty} \int_{B_{4}^{\prime}}\left[\left|\varphi\left(\mathbf{x}^{\prime}\right) \mathbf{v}^{\prime}\right|^{2} \dot{\vartheta}\left(t^{\prime}\right)+\nu\left|\mathbf{v}^{\prime}\right|^{2} \Delta^{\prime} \varphi^{2}\left(\mathbf{x}^{\prime}\right) \vartheta\left(t^{\prime}\right)+\left(\left|\mathbf{v}^{\prime}\right|^{2}+2 p^{\prime}\right) \mathbf{v}^{\prime} \cdot \nabla^{\prime} \varphi^{2}\left(\mathbf{x}^{\prime}\right) \vartheta\left(t^{\prime}\right)\right. \\
\left.+\frac{1}{2} a\left|\varphi\left(\mathbf{x}^{\prime}\right) \mathbf{v}^{\prime}\right|^{2} \vartheta\left(t^{\prime}\right)+\frac{1}{2} a\left(\mathbf{x}^{\prime} \cdot \nabla^{\prime} \varphi^{2}\left(\mathbf{x}^{\prime}\right)\right)\left|\mathbf{v}^{\prime}\right|^{2} \vartheta\left(t^{\prime}\right)\right] \mathrm{d} \mathbf{x}^{\prime} \mathrm{d} t^{\prime}
\end{gathered}
$$

Choosing $\vartheta\left(t^{\prime}\right)=\mathrm{e}^{-\frac{2}{3} a\left(t^{\prime}-t_{\delta}^{\prime}\right)}\left[\mathcal{R}_{1 / m} \chi\right]\left(t^{\prime}\right)$, where $\chi$ is the characteristic function of the interval $\left(t_{\delta}^{\prime}, t^{\prime}\right)$ and $\mathcal{R}_{1 / m}$ is a one-dimensional mollifier with the kernel supported in $(-1 / m, 1 / m)$, and letting $m \rightarrow \infty$, we obtain

$$
\begin{aligned}
& \left\|\varphi \mathbf{v}^{\prime}\left(., t^{\prime}\right)\right\|_{2 ; B_{4}^{\prime}}^{2} \mathrm{e}^{-\frac{2}{3} a\left(t^{\prime}-t_{\delta}^{\prime}\right)}+\frac{a}{6} \int_{t_{\delta}^{\prime}}^{t^{\prime}}\left\|\varphi \mathbf{v}^{\prime}(., \tau)\right\|_{2 ; B_{4}^{\prime}}^{2} \mathrm{e}^{-\frac{2}{3} a\left(\tau-t_{\delta}^{\prime}\right)} \mathrm{d} \tau \\
& +2 \nu \int_{t_{\delta}^{\prime}}^{t^{\prime}}\left\|\varphi \nabla^{\prime} \mathbf{v}^{\prime}(., \tau)\right\|_{2 ; B_{4}^{\prime}}^{2} \mathrm{e}^{-\frac{2}{3} a\left(\tau-t_{\delta}^{\prime}\right)} \mathrm{d} \tau \\
& \leq\left\|\varphi \mathbf{v}^{\prime}\left(., t_{\delta}^{\prime}\right)\right\|_{2 ; B_{4}^{\prime}}^{2}+\int_{t_{\delta}^{\prime}}^{t^{\prime}} \int_{B_{4}^{\prime}}\left[-\nu \nabla^{\prime}\left|\mathbf{v}^{\prime}\right|^{2} \cdot \nabla^{\prime} \varphi^{2}+\left(\left|\mathbf{v}^{\prime}\right|^{2}+2 p^{\prime}\right)\left(\mathbf{v}^{\prime} \cdot \nabla^{\prime} \varphi^{2}\right)\right. \\
& \left.+\left(\frac{1}{2} a \mathbf{x}^{\prime} \cdot \nabla^{\prime} \varphi^{2}\right)\left|\mathbf{v}^{\prime}\right|^{2}\right] \mathrm{d} \mathbf{x}^{\prime} \mathrm{e}^{-\frac{2}{3} a\left(\tau-t_{\delta}^{\prime}\right)} \mathrm{d} \tau .
\end{aligned}
$$

This inequality holds for a.a. $t_{\delta}^{\prime}>2 a^{-1} \ln 4$ and all $t^{\prime} \geq t_{\delta}^{\prime}$. Note that it can also be formally obtained, multiplying (2.10) by $2 \mathbf{v}^{\prime} \varphi^{2} \mathrm{e}^{-\frac{2}{3} a\left(t^{\prime}-t_{\delta}^{\prime}\right)}$ and integrating in $B_{4}^{\prime} \times\left(t_{\delta}^{\prime}, t^{\prime}\right)$. The second term on the left hand side of inequality (2.18) comes from the integral of $\left|\varphi\left(\mathbf{x}^{\prime}\right) \mathbf{v}^{\prime}\right|^{2}\left[\dot{\vartheta}\left(t^{\prime}\right)+\frac{1}{2} a \vartheta\left(t^{\prime}\right)\right]$ on the right hand side of (2.17). Using the formula $\varphi^{2}\left|\nabla^{\prime} \mathbf{v}^{\prime}\right|^{2}=\left|\nabla^{\prime}\left(\varphi \mathbf{v}^{\prime}\right)\right|^{2}-\left|\nabla^{\prime} \varphi^{2}\right|\left|\mathbf{v}^{\prime}\right|^{2}-$ $\frac{1}{2} \nabla^{\prime} \varphi^{2} \cdot \nabla^{\prime}\left|\mathbf{v}^{\prime}\right|^{2}$, we can further rewrite (2.18) as follows:

$$
\begin{aligned}
& \left\|\varphi \mathbf{v}^{\prime}\left(., t^{\prime}\right)\right\|_{2 ; B_{4}^{\prime}}^{2} \mathrm{e}^{-\frac{2}{3} a\left(t^{\prime}-t_{\delta}^{\prime}\right)}+\frac{a}{6} \int_{t_{\delta}^{\prime}}^{t^{\prime}}\left\|\varphi \mathbf{v}^{\prime}(., \tau)\right\|_{2 ; B_{4}^{\prime}}^{2} \mathrm{e}^{-\frac{2}{3} a\left(\tau-t_{\delta}^{\prime}\right)} \mathrm{d} \tau \\
& +2 \nu \int_{t_{\delta}^{\prime}}^{t^{\prime}}\left\|\nabla^{\prime}\left(\varphi \mathbf{v}^{\prime}(., \tau)\right)\right\|_{2 ; B_{4}^{\prime}}^{2} \mathrm{e}^{-\frac{2}{3} a\left(\tau-t_{\delta}^{\prime}\right)} \mathrm{d} \tau \\
& \leq\left\|\varphi \mathbf{v}^{\prime}\left(., t_{\delta}^{\prime}\right)\right\|_{2 ; B_{4}^{\prime}}^{2}+\int_{t_{\delta}^{\prime}}^{t^{\prime}} \int_{B_{4}^{\prime}}\left[2 \nu\left|\nabla^{\prime} \varphi\right|^{2}\left|\mathbf{v}^{\prime}\right|^{2}+\left(\left|\mathbf{v}^{\prime}\right|^{2}+2 p^{\prime}\right)\left(\mathbf{v}^{\prime} \cdot \nabla^{\prime} \varphi^{2}\right)\right. \\
& \left.+\left(\frac{1}{2} a \mathbf{x}^{\prime} \cdot \nabla^{\prime} \varphi^{2}\right)\left|\mathbf{v}^{\prime}\right|^{2}\right] \mathrm{d} \mathbf{x}^{\prime} \mathrm{e}^{-\frac{2}{3} a\left(\tau-t_{\delta}^{\prime}\right)} \mathrm{d} \tau .
\end{aligned}
$$

\section{Estimates of the right hand side of inequality (2.19)}

The right hand side of inequality (2.19) can be estimated from above by the sum of $\left\|\varphi \mathbf{v}^{\prime}\left(., t_{\delta}^{\prime}\right)\right\|_{2 ; B_{4}^{\prime}}^{2}$ and the two terms $K^{I}(\delta), K^{I I}(\delta)$, where 


$$
\begin{aligned}
K^{I}(\delta):=\int_{t_{\delta}^{\prime}}^{\infty} \int_{A_{3,4}^{\prime}}\left(2 \nu\left|\nabla^{\prime} \varphi\right|^{2}\left|\mathbf{v}^{\prime}\right|^{2}+\left|\mathbf{v}^{\prime}\right|^{2}\left|\mathbf{v}^{\prime} \cdot \nabla^{\prime} \varphi^{2}\right|\right. \\
\left.+\left|\frac{1}{2} a \mathbf{x}^{\prime} \cdot \nabla^{\prime} \varphi^{2}\right|\left|\mathbf{v}^{\prime}\right|^{2}\right) \mathrm{d} \mathbf{x}^{\prime} \mathrm{e}^{-\frac{2}{3} a\left(\tau-t_{\delta}^{\prime}\right)} \mathrm{d} \tau \\
K^{I I}(\delta):=\int_{t_{\delta}^{\prime}}^{\infty} \int_{A_{3,4}^{\prime}}\left|2 p^{\prime}\left(\mathbf{v}^{\prime} \cdot \nabla^{\prime} \varphi^{2}\right)\right| \mathrm{d} \mathbf{x}^{\prime} \mathrm{e}^{-\frac{2}{3} a\left(\tau-t_{\delta}^{\prime}\right)} \mathrm{d} \tau .
\end{aligned}
$$

Due to Lemma1, $K^{I}(\delta) \rightarrow 0$ for $\delta \rightarrow 0+. K^{I I}(\delta)$ can be estimated as follows:

$$
\begin{aligned}
& K^{I I}(\delta) \leq C \int_{t_{\delta}^{\prime}}^{\infty} \int_{A_{3,4}^{\prime}}\left|p^{\prime}\right|\left|\mathbf{v}^{\prime}\right| \mathrm{d} \mathbf{x}^{\prime} \mathrm{e}^{-\frac{2}{3} a\left(\tau-t_{\delta}^{\prime}\right)} \mathrm{d} \tau \\
& \leq C\left[\int_{t_{\delta}^{\prime}}^{\infty}\left(\int_{A_{3,4}^{\prime}}\left|\mathbf{v}^{\prime}\right|^{\frac{s}{s-2}} \mathrm{~d} \mathbf{x}^{\prime}\right)^{\frac{r(s-2)}{s}} \mathrm{~d} \tau\right]^{\frac{1}{r}}\left[\int_{t_{\delta}^{\prime}}^{\infty}\left(\int_{A_{3,4}^{\prime}}\left|p^{\prime}\right|^{\frac{s}{2}} \mathrm{~d} \mathbf{x}^{\prime}\right)^{\frac{2}{s} \frac{r}{r-1}} \mathrm{e}^{-\frac{2}{3} \frac{r}{r-1} a\left(\tau-t_{\delta}^{\prime}\right)} \mathrm{d} \tau\right]^{\frac{r-1}{r}} \\
& =c_{1}(\delta) \mathcal{P}^{\frac{r-1}{r}}(\delta),
\end{aligned}
$$

where

$$
\begin{aligned}
& c_{1}(\delta):=C\left[\int_{t_{\delta}^{\prime}}^{\infty}\left(\int_{A_{3,4}^{\prime}}\left|\mathbf{v}^{\prime}\right|^{\frac{s}{s-2}} \mathrm{~d} \mathbf{x}^{\prime}\right)^{\frac{r(s-2)}{s}} \mathrm{~d} \tau\right]^{\frac{1}{r}} \longrightarrow 0 \quad \text { for } \delta \rightarrow 0+\quad \text { (due to Lemma 1) } \\
& \mathcal{P}(\delta):=\int_{t_{\delta}^{\prime}}^{\infty}\left(\int_{A_{3,4}^{\prime}}\left|p^{\prime}\right|^{\frac{s}{2}} \mathrm{~d} \mathbf{x}^{\prime}\right)^{\frac{2}{s} \frac{r}{r-1}} \mathrm{e}^{-\frac{2}{3} \frac{r}{r-1} a\left(\tau-t_{\delta}^{\prime}\right)} \mathrm{d} \tau .
\end{aligned}
$$

In order to estimate $\mathcal{P}(\delta)$, we use the next lemma.

Lemma 2. Let $0<\gamma<1$. Then there exist constants $c_{2}, c_{3}$ and $c_{4}$ so that the inequality

$$
\begin{aligned}
\int_{A_{3,4}^{\prime}}\left|p^{\prime}\right|^{\frac{s}{2}} \mathrm{~d} \mathbf{x}^{\prime} \leq & c_{2}\left(\int_{B_{1}^{\prime}}\left|\mathbf{v}^{\prime}\right|^{2} \mathrm{~d} \mathbf{x}^{\prime}\right)^{\frac{s}{2}}+c_{3} \int_{A_{1, \mathrm{e}^{\prime} t^{\prime} / 2}}\left|\mathbf{v}^{\prime}\right|^{s} \mathrm{~d} \mathbf{x}^{\prime} \\
& +c_{4}\left(\mathrm{e}^{-\frac{3}{2} a t^{\prime}} \int_{A_{\gamma \mathrm{e}^{\prime} t^{\prime} / 2, \mathrm{e}^{a t^{\prime} / 2}}}\left[\left|\mathbf{v}^{\prime}\right|^{2}+\left|p^{\prime}\right|\right] \mathrm{d} \mathbf{x}^{\prime}\right)^{\frac{s}{2}}
\end{aligned}
$$

holds for a.a. $t^{\prime}>2 a^{-1} \ln (4 / \gamma)$.

Proof. Let $\eta$ be an infinitely differentiable cut-off function in $\mathbb{R}^{3}$ such that

$$
\eta\left(\mathbf{x}^{\prime}\right) \begin{cases}=1 & \text { for }\left|\mathbf{x}^{\prime}\right| \leq \gamma \mathrm{e}^{\frac{1}{2} a t^{\prime}} \\ \in[0,1] & \text { for } \gamma \mathrm{e}^{\frac{1}{2} a t^{\prime}}<\left|\mathbf{x}^{\prime}\right| \leq \mathrm{e}^{\frac{1}{2} a t^{\prime}} \\ =0 & \text { for } \mathrm{e}^{\frac{1}{2} a t^{\prime}}<\left|\mathbf{x}^{\prime}\right|\end{cases}
$$

and

$$
\left|\nabla^{\prime} \eta\right| \leq \frac{2}{1-\gamma} \mathrm{e}^{-\frac{1}{2} a t^{\prime}} \quad \text { and } \quad\left|\nabla^{\prime 2} \eta\right| \leq \frac{8}{(1-\gamma)^{2}} \mathrm{e}^{-a t^{\prime}}
$$

Function $\eta$ can be further expressed in the form $\eta_{1}+\eta_{2}$, where both the functions $\eta_{1}$ and $\eta_{2}$ are from $C_{0}^{\infty}\left(\mathbb{R}^{3}\right)$, with values in $[0,1]$, and such that $\eta_{1}=1$ on $B_{1}^{\prime}$ and $\eta_{1}=0$ on $\mathbb{R}^{3} \backslash B_{2}^{\prime}$. Thus, function $\eta_{1}$ is supported in the closure of $B_{2}^{\prime}$ and $\eta_{2}$ is supported in $\mathbb{R}^{3} \backslash B_{1}^{\prime}$. 
The function $\eta p^{\prime}$ satisfies the obvious identity

$$
\eta\left(\mathbf{x}^{\prime}\right) p^{\prime}\left(\mathbf{x}^{\prime}, t^{\prime}\right)=-\frac{1}{4 \pi} \int_{\mathbb{R}^{3}} \frac{1}{\left|\mathbf{x}^{\prime}-\mathbf{y}^{\prime}\right|}\left[\Delta^{\prime}\left(\eta p^{\prime}\right)\right]\left(\mathbf{y}^{\prime}, t^{\prime}\right) \mathrm{d} \mathbf{y}^{\prime}
$$

for $\mathbf{x}^{\prime} \in \mathbb{R}^{3}$. Integrating by parts and using the formula $\Delta^{\prime} p^{\prime}=-\partial_{i}^{\prime} \partial_{j}^{\prime}\left(v_{i}^{\prime} v_{j}^{\prime}\right)$ (which we obtain if we apply operator $\operatorname{div}^{\prime}$ to equation $(2.10)$ ), we derive that

$$
\eta\left(\mathbf{x}^{\prime}\right) p^{\prime}\left(\mathbf{x}^{\prime}, t^{\prime}\right)=p_{1}^{\prime}\left(\mathbf{x}^{\prime}, t^{\prime}\right)+p_{2}^{\prime}\left(\mathbf{x}^{\prime}, t^{\prime}\right)+p_{3}^{\prime}\left(\mathbf{x}^{\prime}, t^{\prime}\right),
$$

where

$$
\begin{aligned}
& p_{1}^{\prime}\left(\mathbf{x}^{\prime}, t^{\prime}\right)=-\frac{1}{4 \pi} \int_{B_{2}^{\prime}} \frac{\partial^{2}}{\partial y_{i}^{\prime} \partial y_{j}^{\prime}}\left(\frac{1}{\left|\mathbf{x}^{\prime}-\mathbf{y}^{\prime}\right|}\right)\left[\eta_{1} v_{i}^{\prime} v_{j}^{\prime}\right]\left(\mathbf{y}^{\prime}, t^{\prime}\right) \mathrm{d} \mathbf{y}^{\prime}, \\
& p_{2}^{\prime}\left(\mathbf{x}^{\prime}, t^{\prime}\right)=-\frac{1}{4 \pi} \int_{A_{1, \mathrm{e}}^{\prime} t^{\prime} / 2} \frac{\partial^{2}}{\partial y_{i}^{\prime} \partial y_{j}^{\prime}}\left(\frac{1}{\left|\mathbf{x}^{\prime}-\mathbf{y}^{\prime}\right|}\right)\left[\eta_{2} v_{i}^{\prime} v_{j}^{\prime}\right]\left(\mathbf{y}^{\prime}, t^{\prime}\right) \mathrm{d} \mathbf{y}^{\prime}, \\
& p_{3}^{\prime}\left(\mathbf{x}^{\prime}, t^{\prime}\right)=\frac{1}{2 \pi} \int_{A_{\gamma \mathrm{e}^{\prime t^{\prime} / 2}, \mathrm{e}^{a t^{\prime} / 2}}} \frac{x_{i}^{\prime}-y_{i}^{\prime}}{\left|\mathbf{x}^{\prime}-\mathbf{y}^{\prime}\right|^{3}}\left(\frac{\partial \eta}{\partial y_{j}^{\prime}} v_{i}^{\prime} v_{j}^{\prime}\right)\left(\mathbf{y}^{\prime}, t^{\prime}\right) \mathrm{d} \mathbf{y}^{\prime} \\
& +\frac{1}{4 \pi} \int_{A_{\gamma \mathrm{e}^{\mathrm{a}^{\prime} t^{\prime} / 2}, \mathrm{e}^{a t^{\prime} / 2}}} \frac{1}{\left|\mathbf{x}^{\prime}-\mathbf{y}^{\prime}\right|}\left(\frac{\partial^{2} \eta}{\partial y_{i}^{\prime} \partial y_{j}^{\prime}} v_{i}^{\prime} v_{j}^{\prime}\right)\left(\mathbf{y}^{\prime}, t^{\prime}\right) \mathrm{d} \mathbf{y}^{\prime} \\
& +\frac{1}{4 \pi} \int_{A_{\gamma \mathrm{e}^{a t^{\prime} / 2}, \mathrm{e}^{a t^{\prime} / 2}}} \frac{x_{i}^{\prime}-y_{i}^{\prime}}{\left|\mathbf{x}^{\prime}-\mathbf{y}^{\prime}\right|^{3}}\left(\frac{\partial \eta}{\partial y_{i}^{\prime}} p^{\prime}\right)\left(\mathbf{y}^{\prime}, t^{\prime}\right) \mathrm{d} \mathbf{y}^{\prime} \\
& +\frac{1}{4 \pi} \int_{A_{\gamma \mathrm{e}^{a t^{\prime} / 2}, \mathrm{e}^{a t^{\prime} / 2}}} \frac{1}{\left|\mathbf{x}^{\prime}-\mathbf{y}^{\prime}\right|}\left[\Delta^{\prime} \eta p^{\prime}\right]\left(\mathbf{y}^{\prime}, t^{\prime}\right) \mathrm{d} \mathbf{y}^{\prime} .
\end{aligned}
$$

If $\mathbf{x}^{\prime} \in A_{3,4}^{\prime}$ then

$$
\begin{aligned}
& \left|p_{1}^{\prime}\left(\mathbf{x}^{\prime}, t^{\prime}\right)\right| \leq C \int_{B_{2}^{\prime}}\left|\mathbf{v}^{\prime}\right|^{2} \mathrm{~d} \mathbf{y}^{\prime} \leq C \int_{B_{1}^{\prime}}\left|\mathbf{v}^{\prime}\right|^{2} \mathrm{~d} \mathbf{y}^{\prime}+C\left(\int_{A_{1,2}^{\prime}}\left|\mathbf{v}^{\prime}\right|^{s} \mathrm{~d} \mathbf{y}^{\prime}\right)^{\frac{2}{s}}, \\
& \left|p_{3}^{\prime}\left(\mathbf{x}^{\prime}, t^{\prime}\right)\right| \leq C \mathrm{e}^{-\frac{3}{2} a t^{\prime}} \int_{A_{\gamma \mathrm{e}^{\prime} t^{\prime} / 2, \mathrm{e}^{a t^{\prime} / 2}}}\left[\left|\mathbf{v}^{\prime}\right|^{2}+\left|p^{\prime}\right|\right] \mathrm{d} \mathbf{x}^{\prime} .
\end{aligned}
$$

Furthermore, applying the Calderon-Zygmund theorem, we obtain

$$
\int_{A_{1, \mathrm{e}^{a} t^{\prime} / 2}}\left|p_{2}^{\prime}\left(\mathbf{y}^{\prime}, t^{\prime}\right)\right|^{\frac{s}{2}} \mathrm{~d} \mathbf{y}^{\prime} \leq C \int_{A_{1, \mathrm{e}^{\prime} t^{\prime} / 2}}\left|\mathbf{v}^{\prime}\left(\mathbf{y}^{\prime}, t^{\prime}\right)\right|^{s} \mathrm{~d} \mathbf{y}^{\prime} .
$$

Inequalities (3.4)-(3.6) imply (3.2).

Using Lemma 2 we can now estimate $\mathcal{P}(\delta)$ as follows:

$$
\begin{aligned}
\mathcal{P}(\delta) \leq & c_{2} \int_{t_{\delta}^{\prime}}^{\infty}\left(\int_{B_{1}^{\prime}}\left|\mathbf{v}^{\prime}\right|^{2} \mathrm{~d} \mathbf{x}^{\prime}\right)^{\frac{r}{r-1}} \mathrm{e}^{-\frac{2}{3} \frac{r}{r-1} a\left(t^{\prime}-t_{\delta}^{\prime}\right)} \mathrm{d} t^{\prime} \\
& +c_{3} \int_{t_{\delta}^{\prime}}^{\infty}\left(\int_{A_{1, \mathrm{e}^{\prime} t^{\prime} / 2}}\left|\mathbf{v}^{\prime}\right|^{s} \mathrm{~d} \mathbf{x}^{\prime}\right)^{\frac{2}{s} \frac{r}{r-1}} \mathrm{e}^{-\frac{2}{3} \frac{r}{r-1} a\left(t^{\prime}-t_{\delta}^{\prime}\right)} \mathrm{d} t^{\prime}
\end{aligned}
$$

10 


$$
+c_{4} \int_{t_{\delta}^{\prime}}^{\infty}\left(\mathrm{e}^{-\frac{3}{2} a t^{\prime}} \int_{A_{\gamma \mathrm{e}^{\prime t^{\prime} / 2}, \mathrm{e}^{a t^{\prime} / 2}}^{\prime}}\left[\left|\mathbf{v}^{\prime}\right|^{2}+\left|p^{\prime}\right|\right] \mathrm{d} \mathbf{x}^{\prime}\right)^{\frac{r}{r-1}} \mathrm{e}^{-\frac{2}{3} \frac{r}{r-1} a\left(t^{\prime}-t_{\delta}^{\prime}\right)} \mathrm{d} t^{\prime} .
$$

Applying inequality (2.19), we deduce that the first term on the right hand side of (3.7) is

$$
\begin{aligned}
& \leq c_{2}\left[\underset{t^{\prime}>t_{\delta}^{\prime}}{\operatorname{essup}} \int_{B_{1}^{\prime}}\left|\mathbf{v}^{\prime}\right|^{2} \mathrm{~d} \mathbf{x} \mathrm{e}^{-\frac{2}{3} a\left(t^{\prime}-t_{\delta}^{\prime}\right)}\right]^{\frac{r}{r-1}-1} \int_{t_{\delta}^{\prime}}^{\infty} \int_{B_{1}^{\prime}}\left|\mathbf{v}^{\prime}\right|^{2} \mathrm{~d} \mathbf{x}^{\prime} \mathrm{e}^{-\frac{2}{3} a\left(t^{\prime}-t_{\delta}^{\prime}\right)} \mathrm{d} t^{\prime} \\
& \leq \frac{6 c_{2}}{a}\left[\left\|\varphi \mathbf{v}^{\prime}\left(., t_{\delta}^{\prime}\right)\right\|_{2 ; B_{4}^{\prime}}^{2}+K^{I}(\delta)+K^{I I}(\delta)\right]^{\frac{r}{r-1}} .
\end{aligned}
$$

The second term on the right hand hand side of 3.7) equals

$$
\begin{aligned}
& =C \delta^{-\frac{4}{3} \frac{r}{r-1}} \int_{t_{0}-\delta^{2}}^{t_{0}}\left(\int_{\theta(t)<\left|\mathbf{x}-\mathbf{x}_{0}\right|<\sqrt{a} \rho}|\mathbf{v}|^{s} \mathrm{~d} \mathbf{x}\right)^{\frac{2}{s} \frac{r}{r-1}} \theta^{\frac{2 r}{r-1}-\frac{6}{s} \frac{r}{r-1}-2+\frac{4}{3} \frac{r}{r-1}}(t) \mathrm{d} t \\
& =C \delta^{-\frac{4}{3} \frac{r}{r-1}} \int_{t_{0}-\delta^{2}}^{t_{0}}\left(\int_{\theta(t)<\left|\mathbf{x}-\mathbf{x}_{0}\right|<\sqrt{a} \rho}|\mathbf{v}|^{s} \mathrm{~d} \mathbf{x}\right)^{\frac{2}{s} \frac{r}{r-1}} \theta^{\frac{r}{r-1}\left[\frac{2}{r}-\frac{6}{s}+\frac{4}{3}\right]}(t) \mathrm{d} t \\
& \leq C \delta^{-\frac{4}{3} \frac{r}{r-1}}\left[\int_{t_{0}-\delta^{2}}^{t_{0}}\left(\int_{\theta(t)<\left|\mathbf{x}-\mathbf{x}_{0}\right|<\sqrt{a} \rho}|\mathbf{v}|^{s} \mathrm{~d} \mathbf{x}\right)^{\frac{r}{s}} \mathrm{~d} t\right]^{\frac{2}{r-1}}\left[\int_{t_{0}-\delta^{2}}^{t_{0}} \theta^{\frac{r}{r-3}\left[\frac{2}{r}-\frac{6}{s}+\frac{4}{3}\right]}(t) \mathrm{d} t\right]^{\frac{r-3}{r-1}} \\
& =c_{5}(\delta) c_{6}(\delta),
\end{aligned}
$$

where $C=C(a, \rho)$ and

$$
\begin{aligned}
& c_{5}(\delta):=\left[\int_{t_{0}-\delta^{2}}^{t_{0}}\left(\int_{\theta(t)<\left|\mathbf{x}-\mathbf{x}_{0}\right|<\sqrt{a} \rho}|\mathbf{v}|^{s} \mathrm{~d} \mathbf{x}\right)^{\frac{r}{s}} \mathrm{~d} t\right]^{\frac{2}{r-1}} \longrightarrow 0 \text { for } \delta \rightarrow 0+, \\
& c_{6}(\delta):=C \delta^{-\frac{4}{3} \frac{r}{r-1}}\left[\int_{t_{0}-\delta^{2}}^{t_{0}} \theta^{\frac{r}{r-3}\left[\frac{2}{r}-\frac{6}{s}+\frac{4}{3}\right]}(t) \mathrm{d} t\right]^{\frac{r-3}{r-1}}=C \delta^{\frac{2 r}{r-1}\left[1-\frac{2}{r}-\frac{3}{s}\right]}=C .
\end{aligned}
$$

Thus, $c_{5}(\delta) c_{6}(\delta) \rightarrow 0$ as $\delta \rightarrow 0+$. Hence the second term on the right hand hand side of (3.7) tends to zero as $\delta \rightarrow 0+$.

Due to the well known result from [1], saying that the set of singular points of a suitable weak solution has the 1-dimensional Hausdorff measure equal to zero, we can assume (without loss of generality) that $\rho$ and $\gamma$ are such positive numbers that $\mathbf{v}$ and $p$ are bounded on the set $\left\{(\mathbf{x}, t) \in \mathbb{R}^{4} ; \sqrt{a} \gamma \rho<\left|\mathbf{x}-\mathbf{x}_{0}\right|<\sqrt{a} \rho\right.$ and $\left.t_{0}-\rho^{2}<t<t_{0}\right\}$. Then the third term on the right hand side of (3.2) is

$$
\begin{aligned}
& \leq c_{4} \int_{t_{\delta}^{\prime}}^{\infty}\left(\mathrm{e}^{-\frac{3}{2} a t^{\prime}} \int_{A_{\gamma \mathrm{e}^{a t^{\prime} / 2}, \mathrm{e}^{a t^{\prime} / 2}}}\left[\left|\mathbf{v}^{\prime}\right|^{2}+\left|p^{\prime}\right|\right] \mathrm{d} \mathbf{x}^{\prime}\right)^{\frac{r}{r-1}} \mathrm{~d} t^{\prime} \\
& =C \int_{t_{0}-\delta^{2}}^{t_{0}}\left(\int_{\sqrt{a} \gamma \rho<\left|\mathbf{x}-\mathbf{x}_{0}\right|<\sqrt{a} \rho}\left[|\mathbf{v}|^{2}+|p|\right] \mathrm{d} \mathbf{x}\right)^{\frac{r}{r-1}} \theta^{1-\frac{r}{r-1}}(t) \mathrm{d} t \\
& \leq C \int_{t_{0}-\delta^{2}}^{t_{0}} \theta^{1-\frac{r}{r-1}}(t) \mathrm{d} t=: c_{7}(\delta) \longrightarrow 0 \quad \text { as } \delta \rightarrow 0+.
\end{aligned}
$$


Using now (3.1) and estimating $\mathcal{P}(\delta)$ by means of (3.7)- (3.10), we obtain

$$
\begin{aligned}
K^{I I}(\delta) \leq & c_{1}(\delta) \mathcal{P}^{\frac{r-1}{r}}(\delta) \\
\leq & c_{1}(\delta)\left\{\left(\frac{6 c_{2}}{a}\right)^{\frac{r-1}{r}}\left[\left\|\varphi \mathbf{v}^{\prime}\left(., t_{\delta}^{\prime}\right)\right\|_{2 ; B_{4}^{\prime}}^{2}+K^{I}(\delta)+K^{I I}(\delta)\right]\right. \\
& \left.\quad+c_{5}^{\frac{r-1}{r}}(\delta) c_{6}^{\frac{r-1}{r}}(\delta)+c_{7}^{\frac{r-1}{r}}(\delta)\right\} .
\end{aligned}
$$

Assuming that $\delta$ is sufficiently small, the term $c_{1}(\delta)\left(6 c_{2} / a\right)^{\frac{r-1}{r}} K^{I I}(\delta)$ on the right hand side can be absorbed by the left hand side and we get the estimate

$$
K^{I I}(\delta) \leq c_{8}(\delta)\left\{\left(\frac{6 c_{2}}{a}\right)^{\frac{r-1}{r}}\left[\left\|\varphi \mathbf{v}^{\prime}\left(., t_{\delta}^{\prime}\right)\right\|_{2 ; B_{4}^{\prime}}^{2}+K^{I}(\delta)\right]+c_{5}^{\frac{r-1}{r}}(\delta) c_{6}^{\frac{r-1}{r}}(\delta)+c_{7}^{\frac{r-1}{r}}(\delta)\right\},
$$

where

$$
c_{8}(\delta):=\frac{c_{1}(\delta)}{1-c_{1}(\delta)\left(6 c_{2} / a\right)^{\frac{r-1}{r}}} \longrightarrow 0 \quad \text { for } \delta \rightarrow 0+.
$$

Thus, finally, inequality (2.19) yields

$$
\begin{aligned}
& \left\|\varphi \mathbf{v}^{\prime}\left(., t^{\prime}\right)\right\|_{2 ; B_{4}^{\prime}}^{2} \mathrm{e}^{-\frac{2}{3} a\left(t^{\prime}-t_{\delta}^{\prime}\right)}+\frac{a}{6} \int_{t_{\delta}^{\prime}}^{t^{\prime}}\left\|\varphi \mathbf{v}^{\prime}(., \tau)\right\|_{2 ; B_{4}^{\prime}}^{2} \mathrm{e}^{-\frac{2}{3} a\left(\tau-t_{\delta}^{\prime}\right)} \mathrm{d} \tau \\
& \quad+2 \nu \int_{t_{\delta}^{\prime}}^{t^{\prime}}\left\|\nabla^{\prime}\left(\varphi \mathbf{v}^{\prime}(., \tau)\right)\right\|_{2 ; B_{4}^{\prime}}^{2} \mathrm{e}^{-\frac{2}{3} a\left(\tau-t_{\delta}^{\prime}\right)} \mathrm{d} \tau \\
& \leq c_{9}(\delta)\left\|\varphi \mathbf{v}^{\prime}\left(., t_{\delta}^{\prime}\right)\right\|_{2 ; B_{4}^{\prime}}^{2}+c_{10}(\delta)
\end{aligned}
$$

where

$$
\begin{aligned}
c_{9}(\delta) & :=\left[1+c_{8}(\delta)\left(\frac{6 c_{2}}{a}\right)^{\frac{r-1}{r}}\right] \longrightarrow 1 \text { for } \delta \rightarrow 0+ \\
c_{10}(\delta) & :=\left[1+c_{8}(\delta)\left(\frac{6 c_{2}}{a}\right)^{\frac{r-1}{r}}\right] K^{I}(\delta)+c_{8}(\delta)\left[c_{5}^{\frac{r-1}{r}}(\delta) c_{6}^{\frac{r-1}{r}}(\delta)+c_{7}^{\frac{r-1}{r}}(\delta)\right] \\
& \longrightarrow 0 \text { for } \delta \rightarrow 0+
\end{aligned}
$$

In order to control the first term on the right hand side of (3.11), we shall use the next lemma.

Lemma 3. Let function $f$ be integrable and nonnegative on the interval $(0, \infty)$. Let $\zeta>0$. Then at least one of the two statements holds:

$$
\int_{\sigma}^{\infty} f(\tau) \mathrm{e}^{-\frac{2}{3} a(\tau-\sigma)} \mathrm{d} \tau \longrightarrow 0 \quad \text { for } \sigma \rightarrow \infty
$$

(B) there exists a set $\mathcal{E}_{\zeta}^{\prime} \subset(0, \infty)$ such that $m_{1}\left(\mathcal{E}_{\zeta}^{\prime} \cap(\sigma, \infty)\right)>0$ for each $\sigma>0$ (where $m_{1}$ denotes the 1-dimensional Lebesgue measure) and

$$
f(\sigma) \leq \frac{2 a(1+\zeta)}{3} \int_{\sigma}^{\infty} f(\tau) \mathrm{e}^{-\frac{2}{3} a(\tau-\sigma)} \mathrm{d} \tau \quad \text { for } \sigma \in \mathcal{E}_{\zeta}^{\prime}
$$


Proof. Denote $h(\sigma):=\int_{\sigma}^{\infty} f(\tau) \mathrm{e}^{-\frac{2}{3} a \tau} \mathrm{d} \tau$. Then $h^{\prime}(\sigma)=-f(\sigma) \mathrm{e}^{-\frac{2}{3} a \sigma}$ for a.a. $\sigma \in(0, \infty)$. Inequality 3.15) is equivalent to $h^{\prime}(\sigma)+\frac{2}{3} a(1+\zeta) h(\sigma) \geq 0$, which is further equivalent to $\left[\mathrm{e}^{\frac{2}{3} a(1+\zeta) \sigma} h(\sigma)\right]^{\prime} \geq 0$.

Assume that statement (B) does not hold. Then there exists $\sigma_{0}>0$ such that $\left[\mathrm{e}^{\frac{2}{3} a(1+\zeta) \sigma} h(\sigma)\right]^{\prime}$ $<0$ for a.a. $\sigma \in\left(\sigma_{0}, \infty\right)$. Hence $\mathrm{e}^{\frac{2}{3} a(1+\zeta) \sigma} h(\sigma)<\mathrm{e}^{\frac{2}{3} a(1+\zeta) \sigma_{0}} h\left(\sigma_{0}\right)$, i.e.

$$
\mathrm{e}^{\frac{2}{3} a \sigma} h(\sigma)<\mathrm{e}^{-\frac{2}{3} a \zeta \sigma} \mathrm{e}^{\frac{2}{3} a(1+\zeta) \sigma_{0}} h\left(\sigma_{0}\right)
$$

for a.a. $\sigma \in\left(\sigma_{0}, \infty\right)$. Thus, statement (A) holds. The proof of Lemma 3 is completed.

If we apply Lemma 3 with $\sigma=t_{\delta}^{\prime}$ and $f\left(t_{\delta}^{\prime}\right)=\left\|\varphi \mathbf{v}^{\prime}\left(., t_{\delta}^{\prime}\right)\right\|_{2 ; B_{4}^{\prime}}^{2}$, we obtain that either

$$
\int_{t_{\delta}^{\prime}}^{\infty}\left\|\varphi \mathbf{v}^{\prime}(., \tau)\right\|_{2 ; B_{4}^{\prime}}^{2} \mathrm{e}^{-\frac{2}{3} a\left(\tau-t_{\delta}^{\prime}\right)} \mathrm{d} \tau \longrightarrow 0 \text { for } t_{\delta}^{\prime} \rightarrow \infty
$$

or there exists a set $\mathcal{E}_{\zeta}^{\prime} \subset(0, \infty)$ with the properties named in item (B) of Lemma 3 such that

$$
\left\|\varphi \mathbf{v}^{\prime}\left(., t_{\delta}^{\prime}\right)\right\|_{2 ; B_{4}^{\prime}}^{2} \leq \frac{2 a(1+\zeta)}{3} \int_{t_{\delta}^{\prime}}^{\infty}\left\|\varphi \mathbf{v}^{\prime}(., \tau)\right\|_{2 ; B_{4}^{\prime}}^{2} \mathrm{e}^{-\frac{2}{3} a\left(\tau-t_{\delta}^{\prime}\right)} \mathrm{d} \tau \quad \text { for } t_{\delta}^{\prime} \in \mathcal{E}_{\zeta}^{\prime} .
$$

\section{Completion of the proof of Theorem 1 in the case of (3.16)}

In this section, we assume that (3.16) holds. Then there exists a set $\mathcal{G}^{\prime} \subset(0, \infty)$ such that $m_{1}\left(\mathcal{G}^{\prime} \cap\right.$ $(\sigma, \infty))>0$ for each $\sigma>0$ and

$$
\left\|\varphi \mathbf{v}^{\prime}\left(., t_{\delta}^{\prime}\right)\right\|_{2 ; B_{4}^{\prime}} \longrightarrow 0 \quad \text { for } t_{\delta}^{\prime} \in \mathcal{G}, t_{\delta}^{\prime} \rightarrow \infty .
$$

(This can be easily proven by contradiction.) Denote by $\mathcal{G}$ the set of $\delta>0$, corresponding to $t_{\delta}^{\prime} \in \mathcal{G}^{\prime}$, where $\delta$ and $t_{\delta}^{\prime}$ are related by formula (2.7). Inequality (3.11) yields

$$
\begin{gathered}
\left\|\varphi \mathbf{v}^{\prime}\left(., t^{\prime}\right)\right\|_{2 ; B_{4}^{\prime}}^{2} \mathrm{e}^{-\frac{2}{3} a\left(t^{\prime}-t_{\delta}^{\prime}\right)}+\frac{a}{6} \int_{t_{\delta}^{\prime}}^{t^{\prime}}\left\|\varphi \mathbf{v}^{\prime}(., \tau)\right\|_{2 ; B_{4}^{\prime}}^{2} \mathrm{e}^{-\frac{2}{3} a\left(\tau-t_{\delta}^{\prime}\right)} \mathrm{d} \tau \\
+2 \nu \int_{t_{\delta}^{\prime}}^{t^{\prime}}\left\|\nabla^{\prime}\left(\varphi \mathbf{v}^{\prime}(., \tau)\right)\right\|_{2 ; B_{4}^{\prime}}^{2} \mathrm{e}^{-\frac{2}{3} a\left(\tau-t_{\delta}^{\prime}\right)} \mathrm{d} \tau \leq c_{11}(\delta)
\end{gathered}
$$

for $t_{\delta}^{\prime} \in \mathcal{G}^{\prime}$ and $t^{\prime}>t_{\delta}^{\prime}$, where $c_{11}(\delta) \rightarrow 0$ as $\delta \rightarrow 0, \delta \in \mathcal{G}$. Applying now 4.2), we can estimate the integrals on the right hand side of (2.13) in the case when $\delta \in \mathcal{G}$ :

$$
\begin{aligned}
& \int_{t_{\delta}^{\prime}}^{\infty}\left\|\nabla^{\prime}\left(\varphi \mathbf{v}^{\prime}\right)\right\|_{2 ; B_{4}^{\prime}}^{2} \mathrm{e}^{-\frac{2}{3} a\left(t^{\prime}-t_{\delta}^{\prime}\right)} \mathrm{d} t^{\prime} \leq \frac{1}{2 \nu} c_{11}(\delta) \\
& \int_{t_{\delta}^{\prime}}^{\infty}\left\|\varphi \mathbf{v}^{\prime}\right\|_{2 ; B_{4}^{\prime}}^{6} \mathrm{e}^{-2 a\left(t^{\prime}-t_{\delta}^{\prime}\right)} \mathrm{d} t^{\prime} \\
& \quad \leq\left[\operatorname{ess} \sup \left(\left\|\varphi \mathbf{v}^{\prime}\left(., t^{\prime}\right)\right\|_{2 ; B_{4}^{\prime}}^{4} \mathrm{e}^{-\frac{4}{3} a\left(t^{\prime}-t_{\delta}^{\prime}\right)}\right)\right] \int_{t_{n}^{\prime}}^{\infty}\left\|\varphi \mathbf{v}_{\delta}^{\prime}\right\|_{2 ; B_{4}^{\prime}}^{2} \mathrm{e}^{-\frac{2}{3} a\left(t^{\prime}-t_{\delta}^{\prime}\right)} \mathrm{d} t^{\prime} \\
& \quad \leq \frac{6}{a} c_{11}^{3}(\delta) .
\end{aligned}
$$

Since the right hand sides of (4.3) and (4.4) tend to zero for $\delta \rightarrow 0, \delta \in \mathcal{G}$, we obtain (by means of estimate (2.13) that $G^{I I}(\delta) \rightarrow 0$ for the same $\delta$. This, together with (2.4), proves (2.3). Hence $\left(\mathbf{x}_{0}, t_{0}\right)$ is a regular point of solution $\mathbf{v}$. 


\section{Completion of the proof of Theorem 1 in the case of (3.17)}

In this section, we assume that 3.17) holds.

A partition of function $\varphi$. Let $\xi \in(0,1)$. Function $\varphi$ can be expressed in the form $\varphi_{1}^{\xi}+\varphi_{2}^{\xi}$, where both the functions $\varphi_{1}^{\xi}$ and $\varphi_{2}^{\xi}$ are in $C_{0}^{\infty}\left(\mathbb{R}^{3}\right)$,

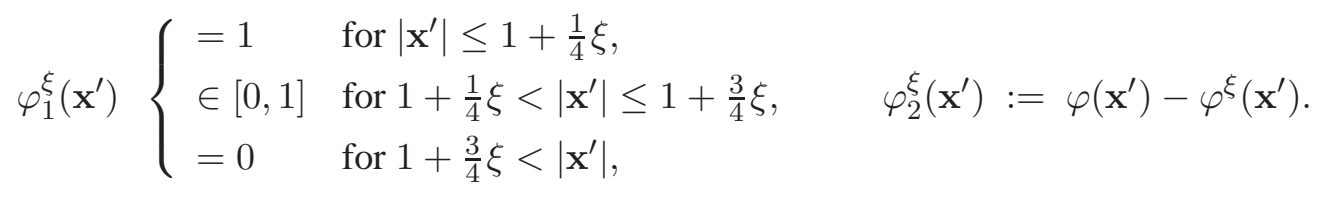

Function $\varphi_{1}^{\xi}$ can be chosen so that $\left|\nabla^{\prime} \varphi_{1}^{\xi}\right| \leq 4 \xi^{-1}$.

A Friedrichs-type estimate of $\varphi_{1}^{\xi} \mathbf{v}^{\prime}$ in $B_{1+\xi}^{\prime}$. Applying the so called Bogovskij operator in $A_{1,1+\xi}^{\prime}$, one can construct a function $\mathbf{w}^{\xi} \in \mathbf{W}_{0}^{1,2}\left(A_{1,1+\xi}^{\prime}\right)$ such that $\operatorname{div}^{\prime} \mathbf{w}^{\xi}=-\nabla^{\prime} \varphi_{1}^{\xi} \cdot \mathbf{v}^{\prime}$ and $\left\|\nabla \mathbf{w}^{\xi}\right\|_{2 ; A_{1,1+\xi}^{\prime}} \leq C \xi^{-1}\left\|\mathbf{v}^{\prime}\right\|_{2 ; A_{1,1+\xi}^{\prime}}$. If we extend function $\mathbf{w}^{\xi}$ by zero to the whole ball $B_{1+\xi}^{\prime}$ then $\varphi_{1}^{\xi} \mathbf{v}^{\prime}-\mathbf{w}^{\xi}$ is divergence-free and belongs to $\mathbf{W}_{0}^{1,2}\left(B_{1+\xi}^{\prime}\right)$. Now we have

$$
\left\|\varphi_{1}^{\xi} \mathbf{v}^{\prime}-\mathbf{w}^{\xi}\right\|_{2 ; B_{1+\xi}^{\prime}} \leq \frac{1+\xi}{\sqrt{\lambda_{S}\left(B_{1}\right)}}\left\|\nabla^{\prime}\left(\varphi_{1}^{\xi} \mathbf{v}^{\prime}-\mathbf{w}^{\xi}\right)\right\|_{2 ; B_{1+\xi}^{\prime}}
$$

(Recall the $\lambda_{S}\left(B_{1}\right)$ is the first eigenvalue of the Dirichlet-Stokesian and $\pi^{2}$ is the principal eigenvalue of the Dirichlet-Laplacian in the unit ball - see Section 1) Hence

$$
\begin{aligned}
\left\|\varphi_{1}^{\xi} \mathbf{v}^{\prime}\right\|_{2 ; B_{1+\xi}^{\prime}} & \leq\left\|\varphi_{1}^{\xi} \mathbf{v}^{\prime}-\mathbf{w}^{\xi}\right\|_{2 ; B_{1+\xi}^{\prime}}+\left\|\mathbf{w}^{\xi}\right\|_{2 ; B_{1+\xi}^{\prime}} \\
& \leq \frac{1+\xi}{\sqrt{\lambda_{S}\left(B_{1}\right)}}\left\|\nabla^{\prime}\left(\varphi_{1}^{\xi} \mathbf{v}^{\prime}-\mathbf{w}^{\xi}\right)\right\|_{2 ; B_{1+\xi}^{\prime}}+\frac{1+\xi}{\pi}\left\|\nabla^{\prime} \mathbf{w}^{\xi}\right\|_{2 ; B_{1+\xi}^{\prime}} \\
& \leq \frac{1+\xi}{\sqrt{\lambda_{S}\left(B_{1}\right)}}\left\|\nabla^{\prime}\left(\varphi_{1}^{\xi} \mathbf{v}^{\prime}\right)\right\|_{2 ; B_{1+\xi}^{\prime}}+\frac{2(1+\xi)}{\pi}\left\|\nabla^{\prime} \mathbf{w}^{\xi}\right\|_{2 ; B_{1+\xi}^{\prime}} \\
& =\frac{1+\xi}{\sqrt{\lambda_{S}\left(B_{1}\right)}}\left\|\nabla^{\prime}\left(\varphi_{1}^{\xi} \mathbf{v}^{\prime}\right)\right\|_{2 ; B_{1+\xi}^{\prime}}+\frac{2(1+\xi)}{\pi}\left\|\nabla^{\prime} \mathbf{w}^{\xi}\right\|_{2 ; A_{1,1+\xi}^{\prime}} \\
& \leq \frac{1+\xi}{\sqrt{\lambda_{S}\left(B_{1}\right)}}\left\|\nabla^{\prime}\left(\varphi_{1}^{\xi} \mathbf{v}^{\prime}\right)\right\|_{2 ; B_{1+\xi}^{\prime}}+\frac{C(1+\xi)}{\xi \pi}\left\|\mathbf{v}^{\prime}\right\|_{2 ; A_{1,1+\xi}^{\prime}}
\end{aligned}
$$

This implies that to each $\xi>0$ and $\kappa>0$ there exists $c_{12}(\kappa, \zeta)>0$ such that

$$
\left\|\varphi_{1}^{\xi} \mathbf{v}^{\prime}\right\|_{2 ; B_{1+\xi}^{\prime}}^{2} \leq \frac{(1+\kappa)(1+\xi)^{2}}{\lambda_{S}\left(B_{1}\right)}\left\|\nabla^{\prime}\left(\varphi_{1}^{\xi} \mathbf{v}^{\prime}\right)\right\|_{2 ; B_{1+\xi}^{\prime}}^{2}+c_{12}(\kappa, \xi)\left\|\mathbf{v}^{\prime}\right\|_{2 ; A_{1,1+\xi}^{\prime}}^{2}
$$

Application of inequality (3.17). The integrand $\left\|\varphi \mathbf{v}^{\prime}\right\|_{2 ; B_{4}^{\prime}}^{2}$ on the right hand side of (3.17) can be expressed in the form

$$
\left\|\varphi \mathbf{v}^{\prime}\right\|_{2 ; B_{4}^{\prime}}^{2}=\left\|\varphi_{1}^{\xi} \mathbf{v}^{\prime}\right\|_{2 ; B_{1+\xi}^{\prime}}^{2}+2\left(\varphi_{1}^{\xi} \mathbf{v}^{\prime}, \varphi_{2}^{\xi} \mathbf{v}^{\prime}\right)_{2 ; A_{1,1+\xi}^{\prime}}+\left\|\varphi_{2}^{\xi} \mathbf{v}^{\prime}\right\|_{2 ; A_{1,4}^{\prime}}^{2}
$$

where

$$
2 \int_{t_{\delta}^{\prime}}^{\infty}\left[\left(\varphi_{1}^{\xi} \mathbf{v}^{\prime}, \varphi_{2}^{\xi} \mathbf{v}^{\prime}\right)_{2 ; A_{1,1+\xi}^{\prime}}+\left\|\varphi_{2}^{\xi} \mathbf{v}^{\prime}\right\|_{2 ; A_{1,4}^{\prime}}^{2}\right] \mathrm{e}^{-\frac{2}{3} a\left(\tau-t_{\delta}^{\prime}\right)} \mathrm{d} \tau=: c_{13}(\delta, \xi) \longrightarrow 0
$$


for $\delta \rightarrow 0+$ for each fixed $\xi$ due to Lemma 1. Substituting inequality (3.17), with the integrand on the right hand side expressed as in (5.3) to 3.11), we obtain

$$
\begin{aligned}
& \left\|\varphi \mathbf{v}^{\prime}\left(., t^{\prime}\right)\right\|_{2 ; B_{4}^{\prime}}^{2} \mathrm{e}^{-\frac{2}{3} a\left(t^{\prime}-t_{\delta}^{\prime}\right)}+\mu \int_{t_{\delta}^{\prime}}^{\infty}\left\|\varphi_{1}^{\xi} \mathbf{v}^{\prime}(., \tau)\right\|_{2 ; B_{1+\xi}^{\prime}}^{2} \mathrm{e}^{-\frac{2}{3} a\left(\tau-t_{\delta}^{\prime}\right)} \mathrm{d} \tau \\
& \quad+2 \nu \int_{t_{\delta}^{\prime}}^{t^{\prime}}\left\|\nabla^{\prime}\left(\varphi \mathbf{v}^{\prime}(., \tau)\right)\right\|_{2 ; B_{4}^{\prime}}^{2} \mathrm{e}^{-\frac{2}{3} a\left(\tau-t_{\delta}^{\prime}\right)} \mathrm{d} \tau \\
& \leq c_{14}(\delta, \zeta, \mu)\left[\int_{t_{\delta}^{\prime}}^{\infty}\left\|\varphi_{1}^{\xi} \mathbf{v}^{\prime}(., \tau)\right\|_{2 ; B_{1+\xi}^{\prime}}^{2} \mathrm{e}^{-\frac{2}{3} a\left(\tau-t_{\delta}^{\prime}\right)} \mathrm{d} \tau+c_{13}(\delta, \xi)\right]+c_{10}(\delta),
\end{aligned}
$$

where $\mu$ is an arbitrary positive number and

$$
c_{14}(\delta, \zeta, \mu):=\frac{2 a(1+\zeta)}{3} c_{9}(\delta)-\frac{a}{6}+\mu \longrightarrow \frac{a}{2}+\mu+\frac{2 a \zeta}{3} \quad \text { as } \delta \rightarrow 0+
$$

(due to (3.12)). Inequality (5.4) is satisfied for $t_{\delta}^{\prime} \in \mathcal{E}_{\zeta}^{\prime}$, for corresponding $\delta=\rho \mathrm{e}^{-\frac{1}{2} a t_{\delta}^{\prime}}$ (we denote by $\mathcal{E}_{\zeta}$ the set of these $\delta$ ) and for all $t^{\prime}>t_{\delta}^{\prime}$. The integrand $\left\|\varphi_{1}^{\xi} \mathbf{v}^{\prime}(., \tau)\right\|_{2 ; B_{1+\xi}^{\prime}}^{2}$ in the integral on the right hand side of (5.4) can be further estimated by means of inequality (5.2). The term $\left\|\nabla^{\prime}\left(\varphi_{1}^{\xi} \mathbf{v}^{\prime}\right)\right\|_{2 ; B_{1+\xi}^{\prime}}^{2}$ on the right hand side of $\underline{5.2)}$ is estimated as follows:

$$
\begin{gathered}
\left\|\nabla^{\prime}\left(\varphi_{1}^{\xi} \mathbf{v}^{\prime}\right)\right\|_{2 ; B_{1+\xi}^{\prime}}^{2}=\left\|\varphi_{1}^{\xi} \nabla^{\prime} \mathbf{v}^{\prime}\right\|_{2 ; B_{1+\xi}^{\prime}}^{2}+2\left(\varphi_{1}^{\xi} \nabla^{\prime} \mathbf{v}^{\prime}, \nabla^{\prime} \varphi_{1}^{\xi} \otimes \mathbf{v}^{\prime}\right)_{2 ; B_{1+\xi}^{\prime}}+\left\|\nabla^{\prime} \varphi_{1}^{\xi} \otimes \mathbf{v}^{\prime}\right\|_{2 ; B_{1+\xi}^{\prime}}^{2} \\
\leq\left\|\nabla^{\prime} \mathbf{v}^{\prime}\right\|_{2 ; B_{1+\xi}^{\prime}}^{2}+\int_{A_{1,1+\xi}^{\prime}} \nabla^{\prime}\left(\varphi_{1}^{\xi}\right)^{2} \cdot \nabla^{\prime} \mathbf{v}^{\prime} \cdot \mathbf{v}^{\prime} \mathrm{d} \mathbf{x}^{\prime}+\left\|\nabla^{\prime} \varphi_{1}^{\xi} \otimes \mathbf{v}^{\prime}\right\|_{2 ; A_{1,1+\xi}^{\prime}}^{2} \\
\leq\left\|\nabla^{\prime}\left(\varphi \mathbf{v}^{\prime}\right)\right\|_{2 ; B_{4}^{\prime}}^{2}-\frac{1}{2} \int_{A_{1,1+\xi}^{\prime}} \Delta^{\prime}\left(\varphi_{1}^{\xi}\right)^{2}\left|\mathbf{v}^{\prime}\right|^{2} \mathrm{~d} \mathbf{x}^{\prime}+\left\|\nabla^{\prime} \varphi_{1}^{\xi} \otimes \mathbf{v}^{\prime}\right\|_{2 ; A_{1,1+\xi}^{\prime}}^{2}
\end{gathered}
$$

Now, inequalities (5.2) and (5.4) yield

$$
\begin{aligned}
& \left\|\varphi \mathbf{v}^{\prime}\left(., t^{\prime}\right)\right\|_{2 ; B_{4}^{\prime}}^{2} \mathrm{e}^{-\frac{2}{3} a\left(t^{\prime}-t_{\delta}^{\prime}\right)}+\mu \int_{t_{\delta}^{\prime}}^{\infty}\left\|\varphi_{1}^{\xi} \mathbf{v}^{\prime}(., \tau)\right\|_{2 ; B_{1+\xi}^{\prime}}^{2} \mathrm{e}^{-\frac{2}{3} a\left(\tau-t_{\delta}^{\prime}\right)} \mathrm{d} \tau \\
& \quad+2 \nu \int_{t_{\delta}^{\prime}}^{t^{\prime}}\left\|\nabla^{\prime}\left(\varphi \mathbf{v}^{\prime}(., \tau)\right)\right\|_{2 ; B_{4}^{\prime}}^{2} \mathrm{e}^{-\frac{2}{3} a\left(\tau-t_{\delta}^{\prime}\right)} \mathrm{d} \tau \\
& \leq c_{14}(\delta, \zeta, \mu) \frac{(1+\kappa)(1+\xi)^{2}}{\lambda_{S}\left(B_{1}\right)} \int_{t_{\delta}^{\prime}}^{\infty}\left\|\nabla^{\prime}\left(\varphi \mathbf{v}^{\prime}(., \tau)\right)\right\|_{2 ; B_{4}^{\prime}}^{2} \mathrm{e}^{-\frac{2}{3} a\left(\tau-t_{\delta}^{\prime}\right)} \mathrm{d} \tau \\
& \quad+c_{14}(\delta, \zeta, \mu) \frac{(1+\kappa)(1+\xi)^{2}}{\lambda_{S}\left(B_{1}\right)} c_{15}(\delta, \kappa, \xi)+c_{14}(\delta, \zeta, \mu) c_{13}(\delta, \xi)+c_{10}(\delta),
\end{aligned}
$$

where

$$
\begin{aligned}
c_{15}(\delta, \kappa, \xi):= & \int_{t_{\delta}^{\prime}}^{\infty}\left[\frac{1}{2} \int_{A_{1,1+\xi}^{\prime}}\left|\Delta^{\prime}\left(\varphi_{1}^{\xi}\right)^{2}\right|\left|\mathbf{v}^{\prime}\right|^{2} \mathrm{~d} \mathbf{x}^{\prime}+\left\|\nabla^{\prime} \varphi_{1}^{\xi} \otimes \mathbf{v}^{\prime}\right\|_{2 ; A_{1,1+\xi}^{\prime}}^{2}\right] \mathrm{e}^{-\frac{2}{3} a\left(t^{\prime}-t_{\delta}^{\prime}\right)} \mathrm{d} t^{\prime} \\
& +c_{12}(\kappa, \xi) \int_{t_{\delta}^{\prime}}^{\infty}\left\|\mathbf{v}^{\prime}\right\|_{2 ; A_{1,1+\xi}^{\prime}}^{2} \mathrm{e}^{-\frac{2}{3} a\left(t^{\prime}-t_{\delta}^{\prime}\right)} \mathrm{d} t^{\prime} .
\end{aligned}
$$


Due to (2.14), $c_{15}(\delta, \kappa, \xi) \rightarrow 0$ for all fixed $\kappa, \xi$ and $\delta \rightarrow 0+$. We observe from (5.5) and from the inequality $a<4 \nu \lambda_{S}\left(B_{1}\right)$ (see the assumptions of Theorem 10 that there exist positive numbers $\delta_{0}, \xi, \zeta, \mu, \kappa$ and $\epsilon$ such that

$$
2 \nu-c_{14}(\delta, \zeta, \mu) \frac{(1+\kappa)(1+\xi)^{2}}{\lambda_{S}\left(B_{1}\right)} \geq \epsilon
$$

for all $0<\delta \leq \delta_{0}$. Then inequality (5.7), with these fixed numbers $\xi, \zeta, \mu \kappa$ and $\epsilon$, and with $\delta \in \mathcal{E}_{\zeta}, 0<\delta \leq \delta_{0}$, yields

$$
\begin{aligned}
& \left\|\varphi \mathbf{v}^{\prime}\left(., t^{\prime}\right)\right\|_{2 ; B_{4}^{\prime}}^{2} \mathrm{e}^{-\frac{2}{3} a\left(t^{\prime}-t_{\delta}^{\prime}\right)}+\mu \int_{t_{\delta}^{\prime}}^{\infty}\left\|\varphi_{1}^{\xi} \mathbf{v}^{\prime}(., \tau)\right\|_{2 ; B_{1+\xi}^{\prime}}^{2} \mathrm{e}^{-\frac{2}{3} a\left(\tau-t_{\delta}^{\prime}\right)} \mathrm{d} \tau \\
& \quad+\epsilon \int_{t_{\delta}^{\prime}}^{t^{\prime}}\left\|\nabla^{\prime}\left(\varphi \mathbf{v}^{\prime}(., \tau)\right)\right\|_{2 ; B_{4}^{\prime}}^{2} \mathrm{e}^{-\frac{2}{3} a\left(\tau-t_{\delta}^{\prime}\right)} \mathrm{d} \tau \\
& \leq c_{14}(\delta, \zeta, \mu) \frac{(1+\kappa)(1+\xi)^{2}}{\lambda_{S}\left(B_{1}\right)} c_{15}(\delta, \kappa, \xi)+c_{14}(\delta, \zeta, \mu) c_{13}(\delta, \xi)+c_{10}(\delta) .
\end{aligned}
$$

Thus, we deduce that an analogous expression to the left hand side of (4.2) tends to zero as $\delta \rightarrow 0$, $\delta \in \mathcal{E}_{\zeta}$. The proof of Theorem 1 can now be completed in the same way as in Section 4 after (4.2).

\section{A generalization of Theorem 1}

The assumption $a<4 \nu \lambda_{S}\left(B_{1}\right)$ in Theorem 1 represents a restriction on the shape of paraboloid $P_{a}$ : the paraboloid cannot be arbitrarily wide and set $U_{a, \rho}$ (where $\mathbf{v}$ is supposed to satisfy the Serrin-type condition, considered for fixed $\rho$ ) therefore cannot be arbitrarily small. The condition $a<4 \nu \lambda_{S}\left(B_{1}\right)$ is used only in Section 5 , where it guarantees the validity of inequality (5.8). There arises a natural question whether Theorem 1 can be improved so that the Serrin-type integrability condition would be assumed on a smaller set than $U_{a, \rho}$. We present such a possibility in this section.

In order to stress the dependence on parameter $a$, we further denote the function $\theta(t) \equiv$ $\sqrt{a\left(t_{0}-t\right)}$ by $\theta_{a}(t)$. Then $\theta_{1}(t)=\sqrt{t_{0}-t}$. Note that set $U_{a, \rho}$ (see Section 11) can also be defined as follows:

$$
U_{a, \rho}=\left\{(\mathbf{x}, t) \in \mathbb{R}^{4} ; t_{0}-\rho^{2}<t<t_{0}, \mathbf{x} \in B_{\sqrt{a} \rho}\left(\mathbf{x}_{0}\right) \backslash \overline{\left[\mathbf{x}_{0}+\theta_{1}(t) B_{\sqrt{a}}(\mathbf{0})\right]}\right\} .
$$

Furthermore, since the least eigenvalue of the Dirichlet-Stokes operator in $B_{\sqrt{a}}$ (any ball in $\mathbb{R}^{3}$ with the radius $\sqrt{a})$ is $\lambda_{S}\left(B_{\sqrt{a}}\right)=\lambda_{S}\left(B_{1}\right) / a$, the condition $a<4 \nu \lambda_{S}\left(B_{1}\right)$ is equivalent to $1<4 \nu \lambda_{S}\left(B_{\sqrt{a}}\right)$.

These notes lead us to the generalization of Theorem 1 assume that $D$ is a bounded open set in $\mathbb{R}^{3}$ (not necessarily connected), with a Lipschitzian boundary and containing point $\mathbf{0}$. Let $\lambda_{S}(D)$ be the least eigenvalue of the Dirichlet-Stokes operator in $D$. We define set $U_{\rho}$ (analogous to the previous $U_{a, \rho}$ ) to be

$$
U_{\rho}:=\left\{(\mathbf{x}, t) \in \mathbb{R}^{4} ; t_{0}-\rho<t<t_{0}, \mathbf{x} \in B_{\rho}\left(\mathbf{x}_{0}\right) \backslash \overline{\left[\mathbf{x}_{0}+\theta_{1}(t) D\right]}\right\} .
$$

Now we can formulate the theorem: 
Theorem 2. Let $\mathbf{v}$ be a suitable weak solution of system (1.1), (1.2), $\left(\mathbf{x}_{0}, t_{0}\right) \in Q_{T}$ and $\rho>0$ be so small that $Q_{1, \rho} \subset Q_{T}$. Assume that $D$ is a bounded open set in $\mathbb{R}^{3}$ with a Lipschitzian boundary, containing point $\mathbf{0}$, such that $1<4 \nu \lambda_{S}(D)$ and function $\mathbf{v}$ satisfies the integrability condition in set $U_{\rho}$ :

$$
\int_{t_{0}-\rho^{2}}^{t_{0}}\left(\int_{B_{\rho}\left(\mathbf{x}_{0}\right) \backslash\left[\mathbf{x}_{0}+\theta_{1}(t) D\right]}|\mathbf{v}(\mathbf{x}, t)|^{s} \mathrm{~d} \mathbf{x}\right)^{\frac{r}{s}} \mathrm{~d} t<\infty
$$

for some $r, s$, satisfying inequalities (1.8). Then $\left(\mathbf{x}_{0}, t_{0}\right)$ is a regular point of solution $\mathbf{v}$.

Theorem 2 can be proven in the same way as Theorem 11, up to smaller modifications. The most important ones are: we use function $\theta_{1}$ instead of $\theta_{a}$, we obtain the sets

$$
\begin{aligned}
& V^{\prime}=\left\{\left(\mathbf{x}^{\prime}, t^{\prime}\right) \in \mathbb{R}^{4} ; t^{\prime}>0 \text { and } \mathbf{x}^{\prime} \in D\right\}, \\
& U^{\prime}=\left\{\left(\mathbf{x}^{\prime}, t^{\prime}\right) \in \mathbb{R}^{4} ; t^{\prime}>0,\left|\mathbf{x}^{\prime}\right|<\mathrm{e}^{\frac{1}{2} t^{\prime}}, \mathbf{x}^{\prime} \notin D\right\}
\end{aligned}
$$

instead of $V_{a, \rho}$ and $U_{a, \rho}$, we deal with $U_{\xi}(D)$ (the $\xi$-neighbourhood of $D$ ) instead of $B_{1+\xi}^{\prime}$ and the cut-off function $\varphi$ decreases from one to zero in $A_{R+1, R+2}^{\prime}$ instead of $A_{3,4}^{\prime}$ (where $R$ is so large that $D \subset B_{R}(\mathbf{0})$ ).

Theorem 1 is a special case of Theorem 2 corresponding to the choice $D=B_{\sqrt{a}}(\mathbf{0})$. However, $D$ can generally have another shape than $B_{\sqrt{a}}(\mathbf{0})$ (it can be e.g. "larger" than $B_{\sqrt{a}}(\mathbf{0})$ in some directions) and it can still satisfy the condition $1<4 \nu \lambda_{S}(D)$. The question of dependence of $\lambda_{S}(D)$ on $D$ is discussed in greater detail in [12] and [21].

Acknowledgments. The research was supported by the Grant Agency of the Czech Republic (grant No. 13-00522S) and by the Academy of Sciences of the Czech Republic (RVO 67985840).

\section{References}

[1] L. Caffarelli, R. Kohn and L. Nirenberg: Partial regularity of suitable weak solutions of the Navier-Stokes equations. Comm. on Pure and Appl. Math. 35, 1982, 771-831.

[2] R. Farwig, H. Kozono and H. Sohr: Criteria of local in time regularity of the Navier-Stokes equations beyond Serrin's condition. Banach Center Publ. 81, Parabolic and Navier-Stokes equations, Part 1, Warsaw 2008, 175-184.

[3] G. P. Galdi: An Introduction to the Navier-Stokes initial-boundary value problem. In Fundamental Directions in Mathematical Fluid Mechanics, ed. G. P. Galdi, J. Heywood, R. Rannacher, series "Advances in Mathematical Fluid Mechanics". Birkhauser, Basel 2000, 1-98.

[4] P. Kučera and Z. Skalák: A note on the generalized energy inequality in the Navier-Stokes equations. Appl. Math. 48, 2003, 537-545.

[5] O. A. Ladyzhenskaya: Mathematical Problems in the Dynamics of Viscous Incompressible Fluid. Gordon and Breach, New York 1963.

[6] O. A. Ladyzhenskaya and G. A. Seregin: On partial regularity of suitable weak solutions to the three-dimensional Navier-Stokes equations. J. Math. Fluid Mech. 1, 1999, 356-387.

[7] F. Lin: A new proof of the Caffarelli-Kohn-Nirenberg theorem. Comm. on Pure and Appl. Math. 51, 1998, 241-257. 
[8] J. Nečas and J. Neustupa: New conditions for local regularity of a suitable weak solution to the Navier-Stokes equations. J. Math. Fluid Mech. 4, 2002, 237-256.

[9] J. Neustupa: A note on local interior regularity of a suitable weak solution to the NavierStokes problem. To appear in Discr. Cont. Dyn. Systems, Ser. S.

[10] J. Neustupa: A removable singularity in a suitable weak solution to the Navier-Stokes equations. Nonlinearity 25, 2012, 1695-1708.

[11] R. S. Saks: Spectral problems for the Curl and Stokes operators. Doklady Mathematics 76, 2, 2007, 724-728.

[12] I. Šebestová and T. Vejchodský: Two-sided bounds for eigenvalues of differential operators with applications to friedrichs, Poincaré, trace and similar constants. Nečas Center for Mathematical Modelling, Preprint No. 2013-05, Prague 2013.

[13] G. Seregin and V. Šverák: On smoothness of suitable weak solutions to the Navier-Stokes equations. J. of Math. Sci. 130, 4, 2005, 4884-4892.

[14] G. A. Seregin: Local regularity for suitable weak solutions of the Navier-Stokes equations. Russian Math. Surveys 62, 3, 2007, 595-614.

[15] H. Sohr: The Navier-Stokes Equations. An Elementary Functional Analytic Approach. Birkhäuser Advanced Texts, Basel-Boston-Berlin 2001.

[16] S. Takahashi: On interior regularity criteria for weak solutions of the Navier-Stokes equations. Manuscripta Math. 69, 3, 1990, 237-254.

[17] G. Talenti: Best constant in Sobolev inequality. Ann. Mat. Pura Appl. 110, 1976, 353-372.

[18] R. Temam: Navier-Stokes Equations. North-Holland, Amsterdam-New York-Oxford 1977.

[19] A. Vasseur: A new proof of partial regularity of solutions to Navier-Stokes equations. Nonlin. Diff. Eq. and Appl. 14, 2007, 753-785.

[20] J. Wolf: A new criterion for partial regularity of suitable weak solutions to the NavierStokes equations. Advances in Mathematical Fluid Mechanics, ed. R. Rannacher, A. Sequeira, Springer, Berlin, 2010, 613-630.

[21] V. V. Yurinsky: A lower bound for the principal eigenvalue of the Stokes operator in a random domain. Annales de l'Institut Henri Poincaré - Probabilités et Statistiques 44, 1, 2008, 1-18.

Author's address: $\quad$ Institute of Mathematics, Academy of Sciences of the Czech Republic

Žitná 25, 11567 Praha 1

Czech Republic

e-mail: neustupa@math.cas.cz 